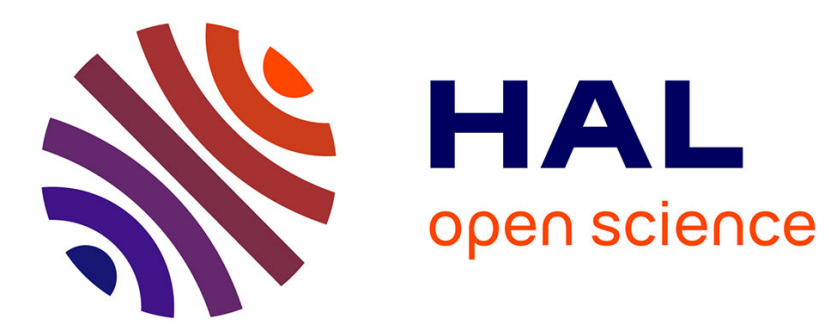

\title{
Closed-locked and Apo-resting State Structures of the Human $\alpha 7$ Nicotinic Receptor: a Computational Study
}

Letizia Chiodo, Thérèse Malliavin, Sergio Giuffrida, Luca Maragliano, Grazia Cottone

\section{- To cite this version:}

Letizia Chiodo, Thérèse Malliavin, Sergio Giuffrida, Luca Maragliano, Grazia Cottone. Closed-locked and Apo-resting State Structures of the Human $\alpha 7$ Nicotinic Receptor: a Computational Study. Journal of Chemical Information and Modeling, 2018, 10.1021/acs.jcim.8b00412 . hal-01907201

\section{HAL Id: hal-01907201 https://hal.science/hal-01907201}

Submitted on 8 Nov 2018

HAL is a multi-disciplinary open access archive for the deposit and dissemination of scientific research documents, whether they are published or not. The documents may come from teaching and research institutions in France or abroad, or from public or private research centers.
L'archive ouverte pluridisciplinaire HAL, est destinée au dépôt et à la diffusion de documents scientifiques de niveau recherche, publiés ou non, émanant des établissements d'enseignement et de recherche français ou étrangers, des laboratoires publics ou privés. 


\section{Computational Biochemistry}

Subscriber access provided by INSTITUT PASTEUR

\section{Closed-locked and Apo-resting State Structures of the Human \#7 Nicotinic Receptor: a Computational Study}

Letizia Chiodo, Therese E. Malliavin, Sergio Giuffrida, Luca Maragliano, and Grazia Cottone

J. Chem. Inf. Model., Just Accepted Manuscript • DOI: 10.1021/acs.jcim.8b00412 • Publication Date (Web): 25 Oct 2018

Downloaded from http://pubs.acs.org on October 30, 2018

\section{Just Accepted}

"Just Accepted" manuscripts have been peer-reviewed and accepted for publication. They are posted online prior to technical editing, formatting for publication and author proofing. The American Chemical Society provides "Just Accepted" as a service to the research community to expedite the dissemination of scientific material as soon as possible after acceptance. "Just Accepted" manuscripts appear in full in PDF format accompanied by an HTML abstract. "Just Accepted" manuscripts have been fully peer reviewed, but should not be considered the official version of record. They are citable by the Digital Object Identifier (DOI®). "Just Accepted" is an optional service offered to authors. Therefore, the "Just Accepted" Web site may not include all articles that will be published in the journal. After a manuscript is technically edited and formatted, it will be removed from the "Just Accepted" Web site and published as an ASAP article. Note that technical editing may introduce minor changes to the manuscript text and/or graphics which could affect content, and all legal disclaimers and ethical guidelines that apply to the journal pertain. ACS cannot be held responsible for errors or consequences arising from the use of information contained in these "Just Accepted" manuscripts. 


\title{
Closed-locked and Apo-resting State Structures of the Human $\alpha 7$ Nicotinic Receptor: A Computational Study
}

\author{
Letizia Chiodo, ${ }^{\dagger}$ Thérèse E. Malliavin, ${ }^{\ddagger}$ Sergio Giuffrida,,$\|$ Luca Maragliano, $\$$ \\ and Grazia Cottone*, \\ $\dagger$ †epartment of Engineering, Campus Bio-Medico University of Rome,Via Á. del Portillo \\ 21, 00128 Rome, Italy \\ $\ddagger$ Institut Pasteur and CNRS UMR 3528, Unité de Bioinformatique Structurale, 25-28 rue \\ du Dr Roux, 75015 Paris, France; Centre de Bioinformatique, Biostatistique et Biologie \\ Intégrative, Institut Pasteur and CNRS USR3756, 25-28 rue du Dr Roux, 75015 Paris, \\ France \\ 9Department of Physics and Chemistry, University of Palermo, Viale delle Scienze Ed. 17, \\ 90128 Palermo, Italy \\ $\S$ Center for Synaptic Neuroscience, Istituto Italiano di Tecnologia, Via Morego 30, 16163 \\ Genoa, Italy; \\ IRCCS Ospedale Policlinico San Martino, Largo Rosanna Benzi 10, 16132 Genoa, Italy \\ ||Present address: Direzione Centrale Analisi Merceologica e Laboratori Chimici, Agenzia \\ Dogane e Monopoli, Via Mario Carucci 71, 00143 Rome, Italy \\ E-mail: grazia.cottone@unipa.it
}

\begin{abstract}
Nicotinic acetylcholine receptors, belonging to the Cys-loop super-family of ligand-


gated ion channels (LGICs), are membrane proteins present in neurons and at neuromuscular junctions. They are responsible for signal transmission, and their function is regulated by neurotransmitters, agonists and antagonists drugs. A detailed knowledge of their conformational transition in response to ligand binding is critical to understand the basis of ligand-receptor interaction, in view of new pharmacological approaches to control receptor activity. However, the scarcity of experimentally derived structures of human channels makes this perspective extremely challenging. To contribute overcoming this issue, we have recently reported structural models for the open and the desensitized states of the human $\alpha 7$ nicotinic receptor. Here, we provide all-atom structural models of the same receptor in two different non-conductive states. The first structure, built via homology modeling and relaxed with extensive Molecular Dynamics simulations, represents the receptor bound to the natural antagonist $\alpha$-conotoxin ImI. After comparison with available experimental data and computational models of other eukaryotic LGICs, we deem it consistent with the "closed-locked" state. The second model, obtained with simulations from the spontaneous relaxation of the open, agonist-bound $\alpha 7$ structure after ligand removal, recapitulates the characteristics of the apo-resting state of the receptor. These results add to our previous work on the active and desensitized state conformations, contributing to the structural characterization of the conformational landscape of the human $\alpha 7$ receptor, and suggesting benchmarks to discriminate among conformations found in experiments or in simulations of LGICs. In particular key interactions at the interface between the extracellular domain and the transmembrane domain are identified, that could be critical to the $\alpha 7$ receptor function.

\section{Introduction}

The identification of functional states of channel proteins based on their structure is a key issue in structural biophysics and electrophysiology. In particular, for ligand gated ion channels (LGICs), the univocal determination of quantities to discriminate between conductive 
The structural characterization of different non-conductive states is especially complex, as they all have in common a non-conductive pore in the transmembrane domain (TMD). These states are usually referred to as: $i$ ) closed-locked, with antagonist ligands bound, $i i$ ) resting or apo, without ligands, and iii) desensitized, inactive in presence of bound agonists. Subtle differences in the structure are expected to be determinant in discriminating these different functional states.

A reference structure for the closed-locked state was missing until few years ago, when an anion selective, human glycine binding channel has been crystallized in complex with the toxin antagonist strychnine. ${ }^{20}$ As for the desensitized states, the identification of characterizing features is still argued, as witnessed by the debate over some structures first associated to closed or even open states, and successively designated as desensitized. ${ }^{21122}$ The most recent structures for desensitized states (human heteropentameric $\alpha 4 \beta 2,233$ human homopentameric $\mathrm{GABA}_{A}{ }^{24}$ bound to the agonist benzamidine, GLIC complexed with fatty acids ${ }^{(25}$ ) present a large heterogeneity even in key quantities for conduction, as for example the pore radius. Finally, with regard to the apo-resting state of LGICs in the absence of ligands, the Torpedo nicotinic acetylcholine receptor (nAChR) from cryo-EM ${ }^{[26127}$ has provided a reference for years. Its channel pore is wider than in the closed bacterial receptors ELIC ${ }^{28}$ or GLIC at $\mathrm{pH} 7 .{ }^{29}$ Few years ago, the $\mathrm{GluCl}$ channel structure has been solved in the apo conformation. ${ }^{30}$ Similarly to the bacterial channels, this structure also exhibits a narrow pore. Recently, Molecular Dynamics (MD) simulations investigated the spontaneous relaxation of $\mathrm{GluCl}$ from an active state, whose structure was solved in complex with the positive allosteric modulator ivermectin,, 31 to a structure very similar to the experimental $\mathrm{GluCl}$ apo-resting, after removal of the ligand. ${ }^{[32}$

Among LGICs, the homopentameric $\alpha 7$ nicotinic receptor is of key interest, being mainly 
(although not uniquely) expressed in the mammalian brain, where it is involved in regulation of plasticity, neuronal growth, and differentiation. ${ }^{33}$ It is also observed in cancer cell proliferation and apoptosis mechanisms. ${ }^{34}$ In particular, up-regulation of $\alpha 7$ (growth stimulator) and desensitization of $\alpha 2 \beta 4$ (growth inhibitor) have been correlated to nicotin exposure and to carcinogenesis. $\frac{35}{35}$

We recently provided an open conductive model of the human nAChR $\alpha 7$ bound to the agonist epibatidine, $\stackrel{18}{18}$ built via homology modeling based on the putatively open channel of $\mathrm{GluCl}^{31}$ (for TMD) and on the structure of AChBP bound to epibatidine ${ }^{36 / 37}$ (for the ligand binding domain, LBD). We also characterized an $\alpha 7$ structure in a non-conductive configuration, showing a very low level of pore hydration, despite being still bound to epibatidine.19 Based on a critical comparison with the most recent experimental structures, we proposed the latter as a representative conformation of the desensitized functional state.

To proceed further in the characterization of the human $\alpha 7$ conformational cycle, we investigate here all-atom structural models of two different non-conducting states. First, we provide a representative structure for the closed-locked form of the channel in presence of the antagonist $\alpha$-Conotoxin ImI $\stackrel{38}{[3}$ We performed homology modeling based on high resolution templates for both the LBD and the TMD, and MD simulations in explicit solvent and lipid bilayer to relax the structure. In addition, we study the spontaneous structural relaxation of the channel from both the closed-locked state and the open, ligand-bound state, as a consequence of sudden removal of the drug. In the latter case, an enhanced conformational heterogeneity, combined with a non-conductive channel, are consistent with a structure representative of the apo-resting state of the human $\alpha 7$ receptor. We establish correspondence between structures and functional states based on local and global structural descriptors of quaternary and tertiary rearrangements. In particular, we focus on the interface of LBD with TMD, the TMD pore, the hydration level, and the atomistic details of the hydrogen bonds pattern in the protein. 


\section{Methods}

\section{Homology modeling}

The $\alpha 7 \mathrm{nAChR}$ structure presents a fivefold symmetry, with an extracellular ligand binding domain, a trans-membrane domain, and an intracellular part. In the TMD, the channel pore, where ions permeate, is formed by the alignment of one helix (M2) from each subunit. The pore opens due to the binding of agonist ligands in the orthosteric site located in the LBD in the five subunits (in the following called $\mathrm{P}_{1}, \ldots, \mathrm{P}_{5}$ ), with an allosteric mechanism widely investigated in LGICs. .739

The protocol to build the $\alpha 7 \mathrm{nAChR}$ homology model in the closed state was similar to the one used in Chiodo et al. $\frac{18}{18}$ for the open state. A chimera template model was built using two different PDB structures. For the LBD, we used the Aplysia californica AChBP

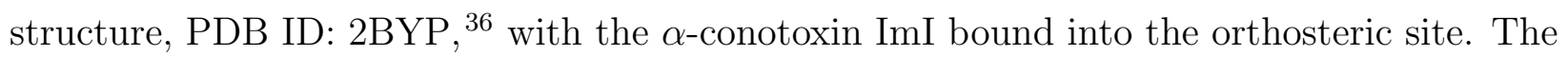
TMD and cys-loop are modelled on the $\mathrm{GluCl}$ in apo-resting conformation, PDB ID: 4TNV, to provide a starting non-conductive configuration for the transmembrane pore. The chains A to $\mathrm{E}$ of $4 \mathrm{TNV}$ and of $2 \mathrm{BYP}$ were superimposed using the backbone of residues 54-61, 76-79, 110-121, 91-93, 141-150 for 4TNV and of residues 55-62, 77-80, 107-118, 91-93, 138-147 for 2BYP. The residues used for the superposition of 4TNV and 2BYP are located in several $\beta$ strands of the extracellular domain, at the principal and complementary faces of the binding site, and not covalently linked to the $\mathrm{C}$ loop. The motivations for this selection are: i) these residues belong to regions relevant for protein function and ii) regions that show very different conformations in the two proteins, as indeed the C loops, are avoided. The chimera was built from the aligned chains using the following fragments: residues 8126 from 2BYP, followed by residues 130-144 from 4TNV corresponding to the Cys-loop, residues 141-203 from 2BYP and finally residues 209-339 from 4TNV corresponding to the 
TMD. The residue numbers were modified to get a continuous numbering from 8 to 335 .

The sequence alignment between $\mathrm{nAChR} \alpha 7$ and the chimera protein ${ }^{18}$ was used along with the $4 \mathrm{TNV} / 2 \mathrm{BYP}$ chimera conformation to calculate an homology model using Modeller 9.10. $\stackrel{42}{ }$ In the sequence alignment (Figure S1 in Supplementary Material), 108 out of the 328 residues of each chain are identical in $\alpha 7$ and in the template sequence and 105 nonidentical residues display homology. As the percentage identity between the target and the template sequences is larger than $30 \%$, the use of homology modeling is appropriate. The script build_model.py provided in the Modeller distribution was used and ten models were produced with the very_slow refinement procedure. The ten models were analyzed with Procheck. $\stackrel{43}{ }$ The model number 10, giving the largest percentage of residues in the core region of the Ramachandran diagram, along with the smallest percentage in the disallowed region and the smallest number of labelled residues, was selected for further analysis. The hydrogens were added using Molprobity $\underline{44}$ and the conformation scored using QMEAN, $\underline{45}$ obtaining a QMEANnorm score of 0.47. For comparison, QMEANnorm scores of 0.55 and 0.59 were obtained for the X-ray crystallographic structures $4 \mathrm{TNV}$ and 2BYP, respectively. The resulting structure is shown in Fig,1,

\section{System set-up and Molecular Dynamics simulations}

Protonation state of ionizable residues at physiological $\mathrm{pH}(7.4)$ is the same as the one of the open channel. $\frac{18 \mid 19}{}$ It was predicted by using the web-based implementation of a method for pKa calculations based on continuum electrostatic model. $\stackrel{46] 47}{ } \mathrm{MD}$ simulations were performed with the NAMD2.12 package, $\frac{4849}{2}$ with the CHARMM27 force field for proteins, ions, and water and the CHARMM36 force field for phospholipids. The protein, complexed with five conotoxin molecules, was embedded in a bilayer/water system. The system set up, the equilibration protocol, and the simulation parameters in the NPT ensemble at T= $310 \mathrm{~K}$ and $\mathrm{P}=1 \mathrm{~atm}$ are as in the case of the active and desensitized state simulations. $18 / 19$ The protein has been inserted into a pre-equilibrated $110 \AA \times 110 \AA$ lipid bilayer originally 

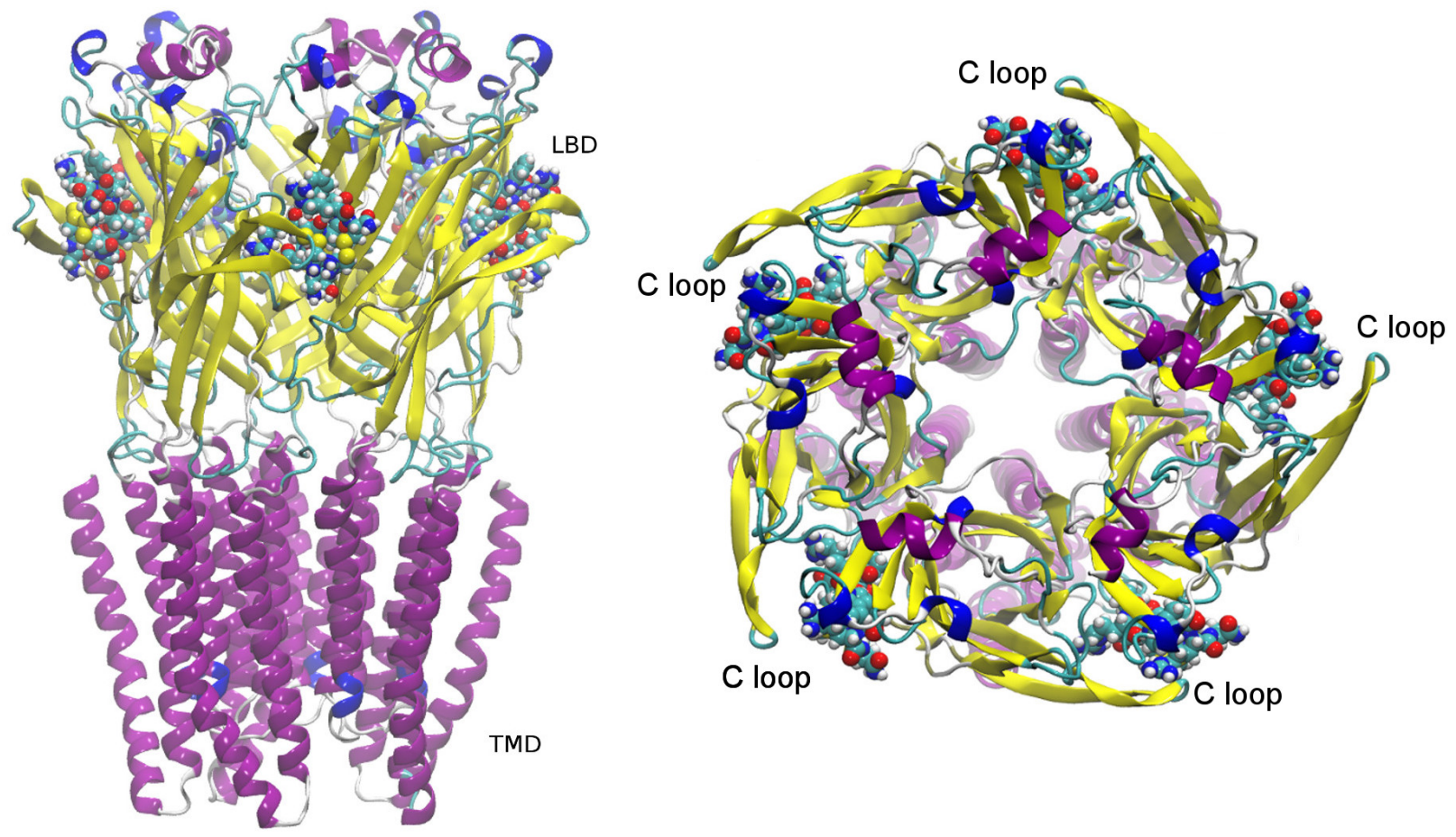

Figure 1: Homology model of the closed-locked $\alpha 7$ bound to $\alpha$-Conotoxin-ImI. Left) side view; right) top view. The protein is in cartoon representation colored according to the secondary structure. The five conotoxin molecules are represented in vdW model. The TMD, LBD and the five $\mathrm{C}$ loops are also indicated. 
composed by 356 palmitoyl-2-oleoyl-sn-glycerol-phosphatidylcholine (POPC) lipids and 5605 waters molecules, obtained from CHARMM-GUI at www . charmm-gui .org. The protein-lipid system underwent 10000 steps of conjugate gradient minimization; the temperature of the system was then increased gradually to $300 \mathrm{~K}$ over $2.4 \mathrm{~ns}$ MD simulation, by a sequence of 200 ps long simulations at constant pressure (1 atm) and temperature, each with an increase of $25 \mathrm{~K}$ and with random reassignment of the atomic velocities at each temperature increase, to let the membrane equilibrate around the protein. At this stage, harmonic positional restraints were applied to all protein atoms, with a force constant of $5 \mathrm{kcal} / \mathrm{mol} / \AA^{2}$. Water was then added to fully solvate the protein/lipid system. The total number of atoms in the system is 143430 (26178 protein atoms plus five conotoxin peptides, 27360 water molecules, 255 POPC lipids). $\mathrm{Na}^{+}$and $\mathrm{Cl}^{-}$ions (157 ions), corresponding to $100 \mathrm{mM}$ solution, were added to neutralize the net system charge. The system was again minimized for 10000 steps; harmonic positional restraints were applied to all lipid and protein atoms with a force constant of $5 \mathrm{kcal} / \mathrm{mol} / \AA^{2}$. The temperature of the system was then increased gradually to $300 \mathrm{~K}$ over $0.6 \mathrm{~ns} \mathrm{MD}$ simulation, by a sequence of $100 \mathrm{ps}$ long simulations at constant pressure (1 atm) and temperature, each with an increase of $50 \mathrm{~K}$ and with random reassignment of the atomic velocities at each temperature increase, to let the water and the ions equilibrate around the protein and the membrane. Further 5 ns were then run in the NPT ensemble at $300 \mathrm{~K}$ and $1 \mathrm{~atm}$, by gradually removing the positional restraints on all protein atoms (force constant from 5 to $1 \mathrm{kcal} / \mathrm{mol} / \AA^{2}, 1$ ns for each force constant value), this time allowing lipids, water and ions to move freely. Periodic boundary conditions were applied, with particle-mesh Ewald long-range electrostatics, $\underline{50}$ using a grid spacing of $1 \AA$ along with a sixth order B-spline charge interpolation scheme. A cutoff of $1.2 \mathrm{~nm}$ for Lennard-Jones potential, with a smooth switching function starting at $1.0 \mathrm{~nm}$, was used. Bonds involving hydrogen atoms were constrained to their equilibrium length using the SHAKE/RATTLE algorithm, $\frac{51}{5 i t h}$ time step of 1 fs.

Starting from equilibrated structures of the open form bound to epibatidine and of the 


\section{Trajectory analysis}

To assess the models for the closed-locked and the apo states, and to compare them with the open active conformation, quaternary and ternary structural descriptors have been selected and monitored along the simulation. Their combination provides a description of the allosteric coupling between the ligand site and the transmembrane ion channel.

Two quaternary changes occurs in the process, specifically a torsional motion of the LBD

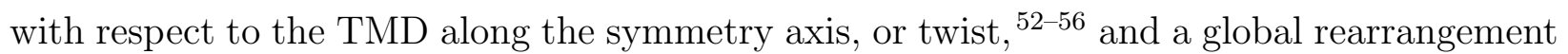
of the entire LBD, called 'blooming' in which the subunits LBD move radially. $230014157+59$ The twist angle is calculated as the angle between the projections of the two vectors from the whole protein center of mass to the center of mass of the LBD and to the center of mass of the TMD (only the $C_{\alpha}$ atoms are considered), respectively, on the plane perpendicular to the symmetry axis of the protein. $\frac{58}{5}$ The blooming is calculated by evaluating the distance of the center of mass of each LBD subunit from the one of the entire LBD, projected on the plane perpendicular to the symmetry axis of the protein.

The quaternary transition in turn involves important changes at the LBD/TMD interface. These are described by the concerted interaction of the M2-M3 loop in the TMD with the Cys-loop and the $\beta 1-\beta 2$ loop in the LBD. $\frac{12[58 / 60] 63}{60}$ To assess the structures modeled in this work, we calculated two distances between i) the $\mathrm{C} \alpha$ atom of Pro256 in the M2-M3 loop and the center of mass of the $\mathrm{C} \alpha$ atoms in the Cys-loop (residues 122-136); ii) the $\mathrm{C} \alpha$ atom of 
Pro256 and the $\mathrm{C} \alpha$ atom of Lys40 in the $\beta 1-\beta 2$ loop. This choice was prompted by careful comparison of our protein primary sequence with other models in the literature. $\frac{58}{5}$

Changes at the LBD-TMD interface induce the re-orientation of the TMD M2 helices with respect to the pore axis. $\frac{12|13| 28|54| 56 \mid 64}{66}$ This is described in terms of the tilt angle that the overall M2 helix ( $C_{\alpha}$ atoms of 23 residues, from Gly231 to Glu253) forms with the protein axis. In the conformational transition the M2 helices undergo both a radial tilting and a tangential motion (polar and azimuthal tilts). These two components are calculated as in Calimet et al. $\stackrel{58}{50}$ use a reference frame centered on the center of mass of the M2 helix. The global $\mathrm{Z}$ axis is set parallel to the main protein symmetry axis (along the channel) and the global $\mathrm{X}$ axis is set as pointing outward the channel, in a radial direction. The polar tilt is defined as the angle between the projection of the helix main (z) axis on the global $\mathrm{XZ}$ plane and the global $\mathrm{Z}$ axis. The azimuthal tilt is defined as the angle between the projection of the helical main (z) axis on the global XY plane and the global X axis. A cartoon schematically representing the helix tilts will be shown along with the plots of the probability distributions of the tilt angles.

Structure and hydration of the channel pore are described by calculating: i) the pore radius, computed using $\mathrm{HOLE}^{\underline{67}}$ in the configuration obtained by averaging over the last 200 ns segment of each trajectory; ii) distances between specific residues of the M2 helices in non-adjacent subunits (crossed distances between the center of mass of residues in facing subunits, for each pair of subunits); iii) the time evolution of the number of water molecules over the last $200 \mathrm{~ns}$ segment of each trajectory, in two regions: the full pore channel, as enclosed by the five M2 helices between Gly231 and Glu253 (30 A long) and in the constriction zone between Leu242 and Leu249 (i.e. Leu9' and Leu16' in prime notation) (10 A long).

The ligand binding site conformation is investigated by calculating the distance between the center of mass of the C-loop ( $C_{\alpha}$ atoms of residues 179-188), capping the ligand, and

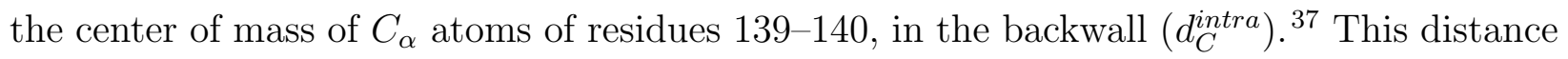
quantifies the degree of $\mathrm{C}$ loop closure around the ligands. 
Moreover, the modes of binding of $\alpha$-conotoxin ImI to our model of the closed-locked human $\alpha 7$ have been investigated. Here we focus our attention on the triad Asp5-Pro6-Arg7 on $\alpha$-conotoxin ImI, experimentally known to be relevant in the interaction of this toxin with the $\alpha 7$ protein. $\stackrel{36 \mid 68}{72}$ Pairwise interactions are described in literature between: i) Asp5 and Arg7 in conotoxin, in concert with ii) interaction of Arg7 with the side chain of Asp197 and the backbone oxygen of Pro196, in the primary subunit; iii) between Arg7 in conotoxin and the side chain of Tyr93 in the primary subunit. These interactions have been evaluated by a hydrogen bond analysis between donor-acceptor pairs in the residues above listed. A hydrogen bond is present if the donor-acceptor distance is shorter than $3.0 \AA$ and the angle between the donor-acceptor axis and the donor-hydrogen bond is less than $30^{\circ}$.

To further characterize the new closed-locked model at atomistic resolution, and with the aim at comparing with the open active conformation, we carried out an analysis of intra-

protein interactions. A donor-acceptor distance shorter than $3.0 \AA$ was defined as "hydrogen bonding" distance. This analysis is aimed at providing a first level filter to establish if donor-acceptor atoms were or not at suitable distance to form a hydrogen bond in one or another protein structure, in this way sieving out some unimportant interactions. "Hydrogen bonding" distances were then filtered according to their presence in only one of the two forms (either in the open-active or in the closed-locked protein structure), at a given moment.

\section{Results}

\section{The closed-locked structure}

\section{Assessment of stability}

To assess the overall stability of the new homology model for the closed-locked conformation, we evaluated the $C_{\alpha}$ Root Mean Square Deviation (RMSD) from the final configuration of the equilibration stage and the Root Mean Square Fluctuations (RMSFs). Results are shown 
in Figure S2 and Figure S3 in Supplementary Material, separately for the LBD and the TMD of the five subunits. The time evolution of the RMSDs points out that, averaging over the five subunits, the conformation of the channel bound to conotoxin is stable on the reported time scale, both at LBD and TMD level. The $C_{\alpha}$ RMSFs profile along the protein chain is quite similar to that obtained from previous simulation of a human receptor homology model based on the putative closed structure of Torpedo marmorata. 11 As for the LBD, the profile is also in good agreement with the one reported for a model of the $\alpha 7$ LBD complexed with $\alpha$-conotoxin ImI. ${ }^{68}$ Peak locations are conserved, in particular, the $\beta 2-\beta 3$ loop, A-loop, Cys-loop, F-loop and the C-loop (that caps the toxin molecules), so as the loops connecting M1-M2 helices in the TMD. We observe a large peak in the region of helix M4 around residue 300, a known consequence of the structural uncertainty associated to the loop M3-M4. ${ }^{73}$ The RMSD curve relative to the subunit P5 (yellow curve) is higher than the others. This behavior is mostly brought about by motions of the $\mathrm{F}$ and $\mathrm{C}$ loops in the subunit, as testified by the corresponding peaks in the RMSFs profiles in Figure S3 (dark yellow curve). Indeed, the RMSD calculated by excluding the $C_{\alpha}$ atoms belonging to the $\mathrm{F}$ and $\mathrm{C}$ loops, appears stationary over time around a value of about $3.2 \AA$ (data not shown). As for the other subunits, the RMSDs calculated according this new selection almost overlap with the RMSDs of the full LBD.

The stability of the closed-locked $\alpha 7$ model at the orthosteric site is evidenced by the distribution of $d_{C}^{\text {intra }}$ shown in Figure S4 in Supplementary Material (dark grey curve). Compared with the epibatidine-bound conformation, $\frac{18}{18}$ a peak is observed at higher values, as expected in the case of a huge molecule as conotoxin ImI. The main peak of the distribution is at $18 \AA$, with a secondary maximum at $19.5 \AA$. Although a shoulder is also observed around $16.7 \AA$, a typical value of the open conformation, overall the degree of the C-loop radial extension is compatible with an antagonist-bound closed state.

The appearance of the secondary peak at $19.5 \AA$ is mostly due to data pertaining to the subunit P5, pointing for a larger conformational freedom of the $\mathrm{C}$ loop in this case, in 
agreement with the corresponding behavior of the LBD RMSD and RMSFs profile above mentioned. Larger fluctuations of $\mathrm{C}$ and F loops in the subunit P5 are correlated with a higher mobility observed for the ligands interacting with this subunit. Indeed, the RMSDs of the two conotoxin molecules at the P5(+)-P4(-) and P1(+)-P5(-) interfaces are higher than the others (data not shown), though all ligands are kept in place during the simulation. Motional heterogeneity among PLGICs subunits is observed also in other computational studies of these proteins. As an example, in a MD simulation of a model of the $\alpha 7$ LBD bound to $\alpha$-conotoxin ImI Yu et al ${ }^{68}$ also obtained considerably higher values of the RMSD and RMSF peaks for one subunit only.

Modes of binding of conotoxin to the ligand pocket residues have been investigated. Figure S5, panel A, shows the ligand/pocket hydrogen bond network in a representative frame of the closed-locked trajectory. Results are in full agreement with results in $\frac{68 \mid 72}{}$. In particular a hydrogen bond is found between Asp5 and Arg7, which has been predicted to stabilize the position of Arg7 and allow it to interact with both Asp 197 and Pro 196. $\underline{68}$ Packing between the side chain of Tyr195 in the primary subunit and the side chain of Arg7 in conotoxin, proposed to explain cation-pi interactions observed in experimental studies, 74 is also apparent. Figure S4, panel B shows the hydrophobic packing of Pro6 by Trp149 and Tyr96, in agreement with the $\alpha$-conotoxin ImI $/ \alpha 7$ model proposed in 6 ; the distances between the center of mass of Pro6 aromatic ring and the center of mass of the aromatic rings in Trp 149 and Tyr96 are $6.5 \AA$ and $6.2 \AA$ respectively. Another bond, reported in $\frac{72}{}$, is found in our model between Asp5 and Tyr188 (not shown), which should allow in turn to stabilize the position of Arg5 in the pocket.

As for the model of the open conformation we refer to in this work, a similar comparison was provided in our previous work. ${ }^{18}$ There, we investigated the pattern of interaction between the ligand pocket residues and epibatidine in our refined model, and demonstrated that they were consistent with the closest available experimental structure, i.e. the X-ray structure of chimera modified toward the human $\alpha 7$ nAChR sequence ${ }^{75}$. 


\section{Quaternary and ternary changes}

In the following, we report the analysis of the quaternary and ternary structural descriptors for the new closed-locked model here proposed, comparing with results on published structures, in particular the open, epibatidine-bound, $\alpha 7$ structure we proposed in Chiodo et al. 18

In LGICs, the process of channel deactivation is associated to a quaternary twist. $\frac{52}{55}$ The twist angle is expected to increase when going from the active towards non conductive states. The distribution of the twist angle values calculated for the closed model is shown in Fig. 2, upper left panel. The protein in the closed structure undergoes indeed a larger

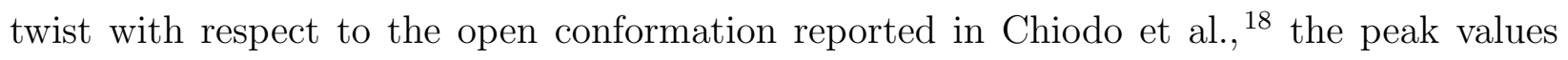
of the distributions being at $25^{\circ}$ and $20^{\circ}$, respectively. This variation is in agreement with results on the GLIC channel, for which a twist increase of $4^{\circ}$ has been observed in closing. $\frac{58}{5}$ Interestingly, the twist in the closed-locked conformation is also more pronounced than in the desensitized state model of $\alpha 7 \cdot \frac{19]}{19}$ Furthermore, the distribution of twist values changes from bimodal in the open conformation (second maximum at $23^{\circ}$ ), to a sharper, almost unimodal distribution in the closed state. This feature is observed in many of the analyzed descriptors of the locked channel. In Fig. 2, upper right panel, the superposition of the TMD in the open and closed structures clearly shows the twist.

The open-to-closed transition involves also a blooming of the $\mathrm{LBD}^{2 / 30|41| 57 / 59}$ in which all LBD subunits move radially outward with respect the channel axis (LBD expansion). The distributions of the blooming distances are shown in Fig. 2, upper middle panel. They are quite overlapped in the closed-locked and open conformations. However, the peak values in the two structures differ of about $1 \AA$, similarly to what obtained by comparing the GLIC channel in the open $(\mathrm{pH} 4.6)$ and closed ( $\mathrm{pH} 7)$ conformations. $\frac{57}{5}$ Slightly different definitions in terms of distances $\frac{57}{57}$ or in terms of reorientation of the $\operatorname{LBD} \beta$ sandwiches are found in literature to describe such collective motion. ${ }^{58}$ However, by consensus a small enlargement of the LBD region has been associated to pore closure. 


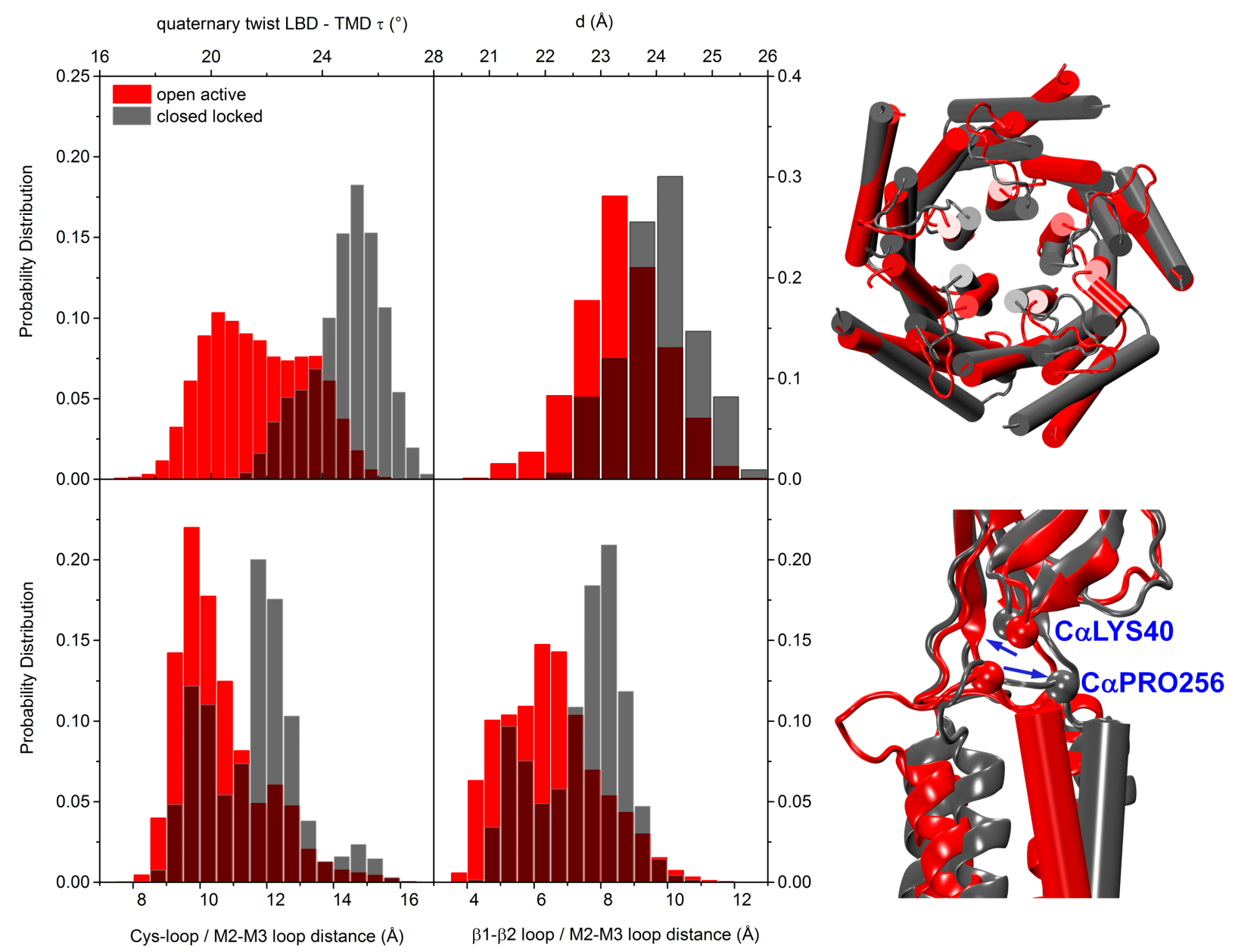

Figure 2: Upper left panel: distribution of twist angles. Data averaged over the five subunits. Upper middle panel: distribution of blooming distances (d). Data collected from the five subunits. Gray bars: closed-locked; red bars: open active. Upper right panel: superposition of the TMD in the open (red) and closed-locked (gray) structures. Lower left panel: distributions of the distances between the $\mathrm{C}_{\alpha}$ atom of Pro256 on the M2-M3 loop and the center of mass of the Cys-loop $\left(C_{\alpha}\right.$ atoms of residues 122-136). Lower middle panel: distributions of the distances between the $\mathrm{C}_{\alpha}$ atom of Pro256 on the M2-M3 loop and the $\mathrm{C}_{\alpha}$ of residue Lys40 on the $\beta 1-\beta 2$ loop. Data collected from the five subunits. Lower right panel: superposition of the entire subunit in the closed-locked (gray) and open (red) structures, one subunit; the $\mathrm{C}_{\alpha}$ atom of Pro256 and the $\mathrm{C}_{\alpha}$ atom of Lys40 are shown in vdW representation colored gray (closed) and red (open). The blue arrows indicate the direction of motions of the $\beta 1-\beta 2$ tip and of the PRO256 in closing. The protein is represented in cartoon.

\section{The LBD/TMD interface}

In LGICs, the quaternary transition induce changes at the LBD/TMD interface. In particular, the M2-M3 loop in the TMD should slide from the Cys-loop towards the $\beta 1-\beta 2$ loop 
to initiate the pore closing. $12 \sqrt{58 \mid 60} \frac{63}{6 n}$ In Fig. 2 the distributions of the distances between the M2-M3 loop and the Cys-loop (lower left panel), or the $\beta 1-\beta 2$ loop (lower middle panel), are shown. In the closed state the main peak of the M2-M3 loop/Cys-loop distance is at $12.5 \AA$, to be compared with $10 \AA$ in the open case, suggesting that the M2-M3 loop is repositioning with respect to the Cys-loop. The distribution of the distances between the M2-M3 loop and the $\beta 1-\beta 2$ tip is broad, reflecting heterogeneous features for the five subunits, but the main peak is clearly shifted by $2 \AA$ with respect to the open form. A similar difference was observed for the closed (strychnine-bound) glycine receptor with respect to

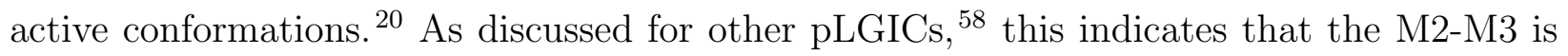
sliding through $\beta 1-\beta 2$ tip, displacing inward towards the ion pore. According to Calimet et al. $\frac{58}{58}$ this conformation change occurs via the up re-position of the $\beta 1-\beta 2$ tip. We observe a similar feature, as shown in Fig. 2, lower right panel, where the blue arrows indicates these putative directions of motion. The concerted motion of the M2M3 tip and the $\beta 1-\beta 2$ tip results in M2M3/ $\beta 1-\beta 2$ distances larger than in the open form. This lets the pore to assume a inverted V-shape as also testified by the behavior of the pore profile shown in the Section below.

\section{M2 helices orientation, pore structure and hydration}

The motion of the M2-M3 loop induces ternary changes in the TMD, and in particular a tilting of the TMD M2 helices toward the pore. $\frac{12[13[28|54| 64] 66}{66}$

The distributions of the tilt angles (both polar and azimuthal components) are shown in Fig. 3. These distributions are broad and bimodal in the active conformation, revealing an asymmetrical, non-concerted motion of the helices. In the closed case, distributions present clear peaks. We observe that the polar tilt changes from positive values in the open channel (main peak at $2.2^{\circ}$ ) to negative values (main peak at $-2^{\circ}$ ) in the closed-locked conformation. This variation confirms that the channel pore shape is changing from a V-shaped to an inverted V-shape pore in the closed channel (see also in Fig. 2, lower right panel). The $4^{\circ}$ 
Figure 3: Left panel: distribution of polar tilt angles. Right panel: distribution of azimuthal tilt angles. Data averaged over the five subunits. Gray bars: closed-locked; red bars: open active. A cartoon is shown in the inset, schematically representing: the protein (gray cylinder) and its principal axis (black line); the M2 helix (magenta cylinder), its principal axis (magenta line) and the radial and lateral tilts (orange and green arrow, respectively).

In Fig. 4, upper panel, we report the pore radius profiles along the protein axis, in the TMD region where the gating occurs. In almost all the pore region, the radius of the closed 
channel is smaller than in the open active case. In particular, the closed conformation is also tighter than the desensitized one at the $16^{\prime}$ position, where one of the hydrophobic rings is located. At the bottom of the pore (towards the intra cellular side, region 100-110 $\AA$ in Fig. 4) the radius is larger than in the open and desensitized conformations modeled, hinting for a inverted $\mathrm{V}$-shape of the pore lumen lined by the M2 helices, as already visible in the tilt angle distribution.

Fig. 4, lower panel, provides a comparison with pore profiles from X-ray structures for different putative closed channels, both $\mathrm{pH}$ gated and ligand gated. We remind that the only closed-locked case so far reported from experiments is a Gly receptor complexed with strychnine. Deep minima at $16^{\prime}, 13^{\prime}, 9^{\prime}$ are present in most of the shown closed structures; the $\alpha 7$ profile is superimposed to that of strychnine channel at positions $20^{\prime}, 13^{\prime}$ and $9^{\prime}$.

In Fig. 5, a representation of the pore through the molecular structure of the closedlocked $\alpha 7$ (left panel) and of the closed Glycine receptor ${ }^{20}$ (middle panel) is shown as a dot surface, along with the pore residues at the constriction points. In $\alpha 7$ a constriction point is also present in the LBD, due to loop conformations extending towards the center of the channel, thus shrinking the pore size.

We remind here that the TMD of closed-locked structure was modeled on the TMD portion of the $\mathrm{GluCl}$ structure in the apo resting state. ${ }^{30}$ The original X-ray structure of the $\mathrm{GluCl}$ apo conformation has a broad constriction region centered at Leu9', plus two less tight constriction regions at $-2^{\prime}$ and $2^{\prime}$, while the pore lumen is wide at $13^{\prime}, 16^{\prime}$ and $20^{\prime}$ (see in Fig. 5, right panel). The modeled $\alpha 7$ structure, at variance, spontaneously relaxes to a conformation where the pore is much more similar to that of the strychnine-bound Gly receptor,confirming the efficiency of the procedure used to build the antagonist-bound model.

A quantitative description of the pore structure at the level of residue rings is provided by the distribution of the crossed distances shown in Figure6. Notably, also for these quantities, the distributions are peaked at very different values in the closed and open conformation. 

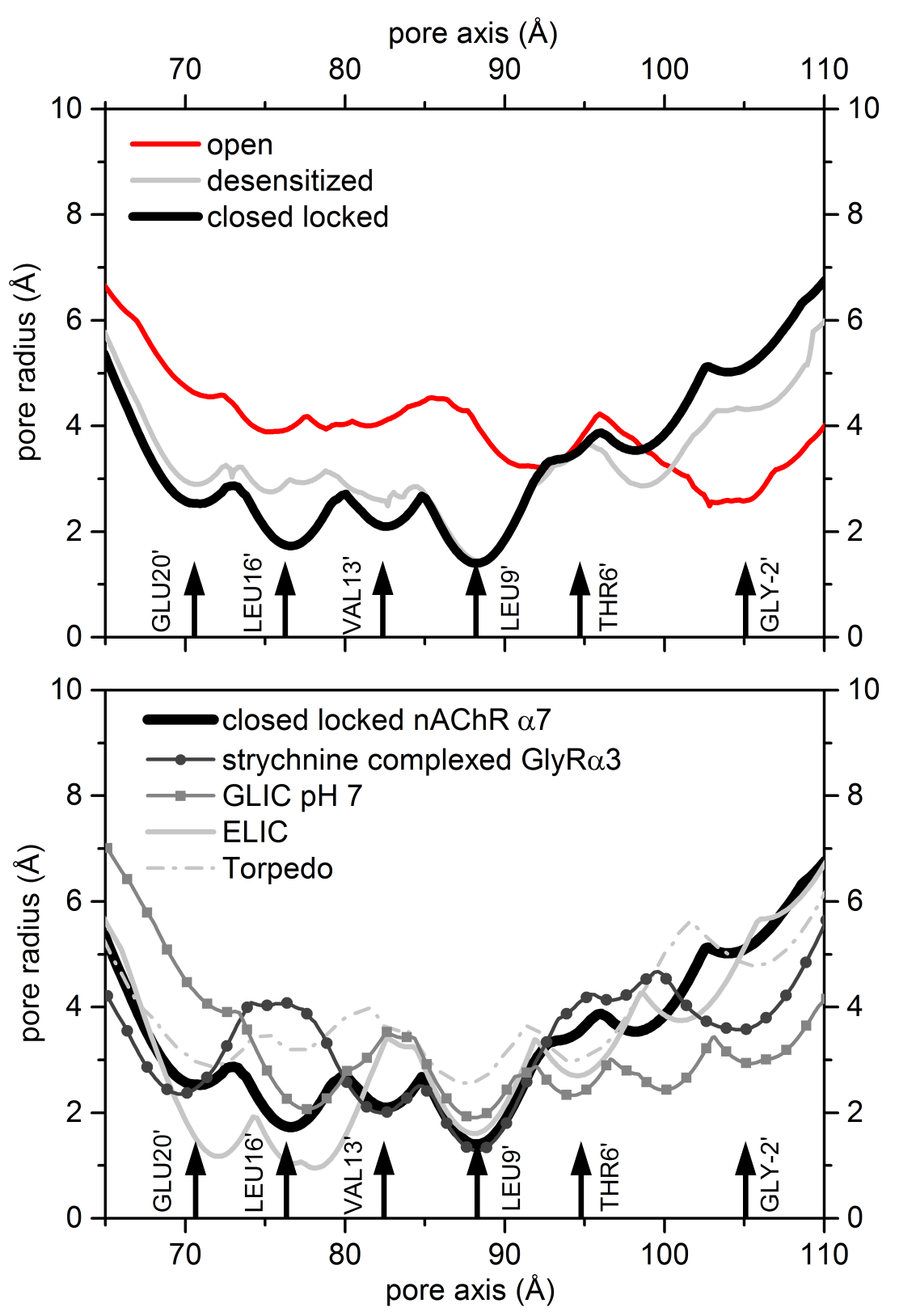

Figure 4: Upper panel: pore radius profiles along the channel axis in the TMD of the modeled $\alpha 7$, in the closed-locked state (black curve), in the desensitized state (gray curve) ${ }^{19}$ and in the open active state (red curve), $\frac{18}{}$ calculated on structures averaged over the last 200 ns of each trajectory. Lower panel: comparison with pore radius profiles for putative closed channels (Torpedo nAChR from cryo-EM, PDB ID: 2BG9; from X-ray: ELIC, PDB ID: 2VL0; GLIC at pH 7, PDB ID: 4NPQ; Glycine receptor bound to strychnine, PDB ID: $5 \mathrm{CFB}$ ). Pore profiles are calculated with HOLE ${ }^{67}$ The black arrows, from left to right, indicate the positions of relevant pore-lining residues of the $\alpha 7$ channel. 

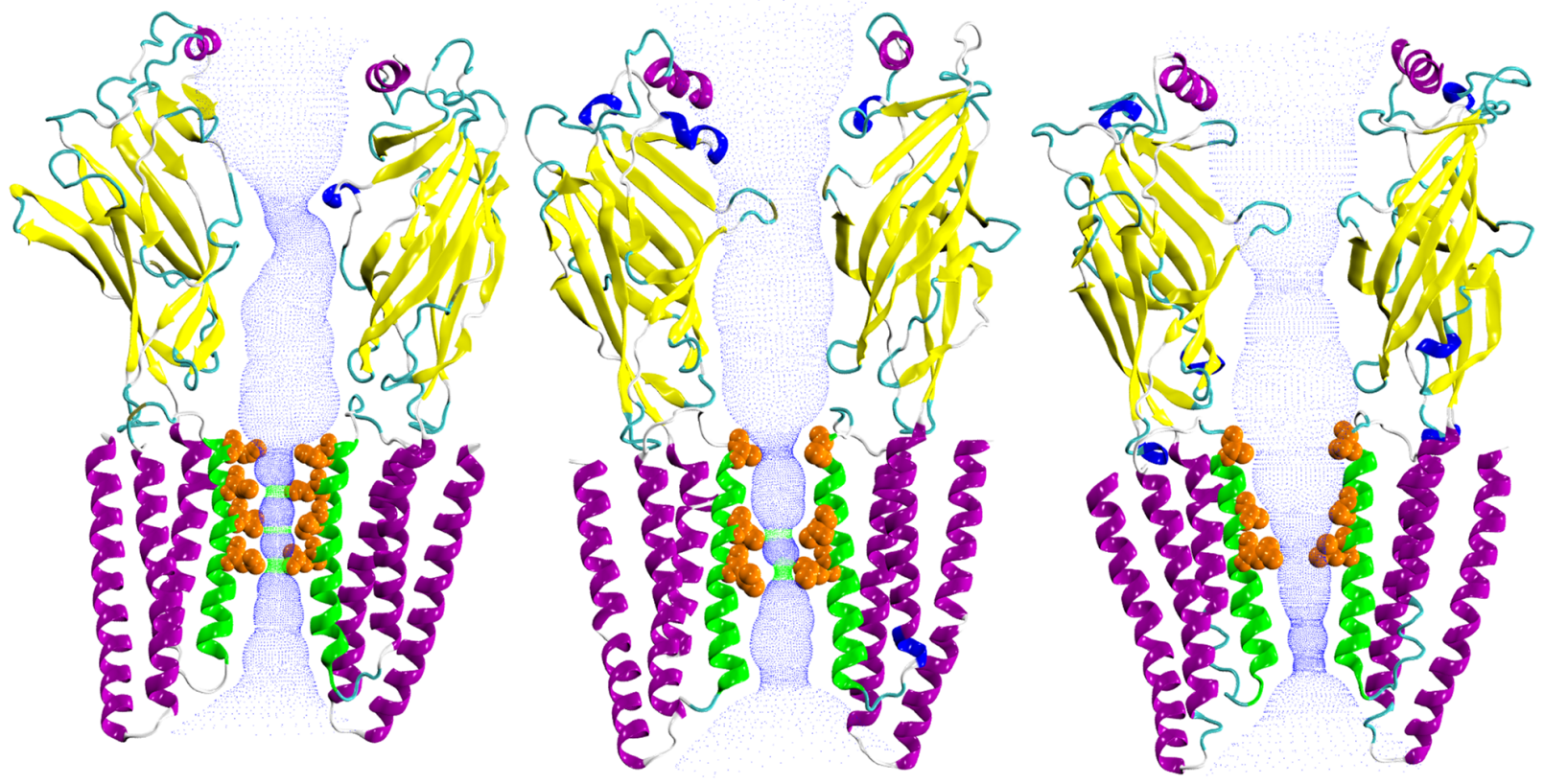

Figure 5: Dot surface of the closed channel pore in the structure averaged along the last $200 \mathrm{~ns}$ portion of the trajectory (left panel); in the X-ray structure of the Glycine receptor bound to strychnine (middle panel, PDB ID: 5CFB); in the X-ray structure of $\mathrm{GluCl}$ apo (right panel, PDB ID: 4TNV). Subunits P1-P3 are sketched in cartoon representation and colored according to the secondary structure. The M2 helices of both subunits are colored in green. Residues at the constriction points are highlighted in vdW representation and colored in orange. From top to bottom: $20^{\prime}, 16^{\prime}, 13^{\prime}, 9^{\prime}$, in the $\alpha 7$ channel; $20^{\prime}, 13^{\prime}, 9^{\prime}$ in the Gly and $\mathrm{GluCl}$ apo receptors, respectively. In the surface, green and blue dots indicate where the pore radius allows room for a single water and where the radius is double the minimum for a single water, respectively. $\underline{67}$

As already observed in the case of other structural quantities, distributions of key distances are extremely sharp in the locked structure, denoting less conformational heterogeneity. The highly localized and peaked feature of the probability distribution in the closed state is a clear fingerprint of a structure with very small fluctuations and variability. Conversely, in the open state, the broadening of the distributions reflects the heterogeneity of conformations needed for function.

The extent of hydration of the pore is strongly correlated to the permeation of ions, hence to receptor's function. Indeed, ion conduction can occur only through hydrated pores. In Fig. 7 we show the time series of the number of water molecules inside the TMD pore, and 

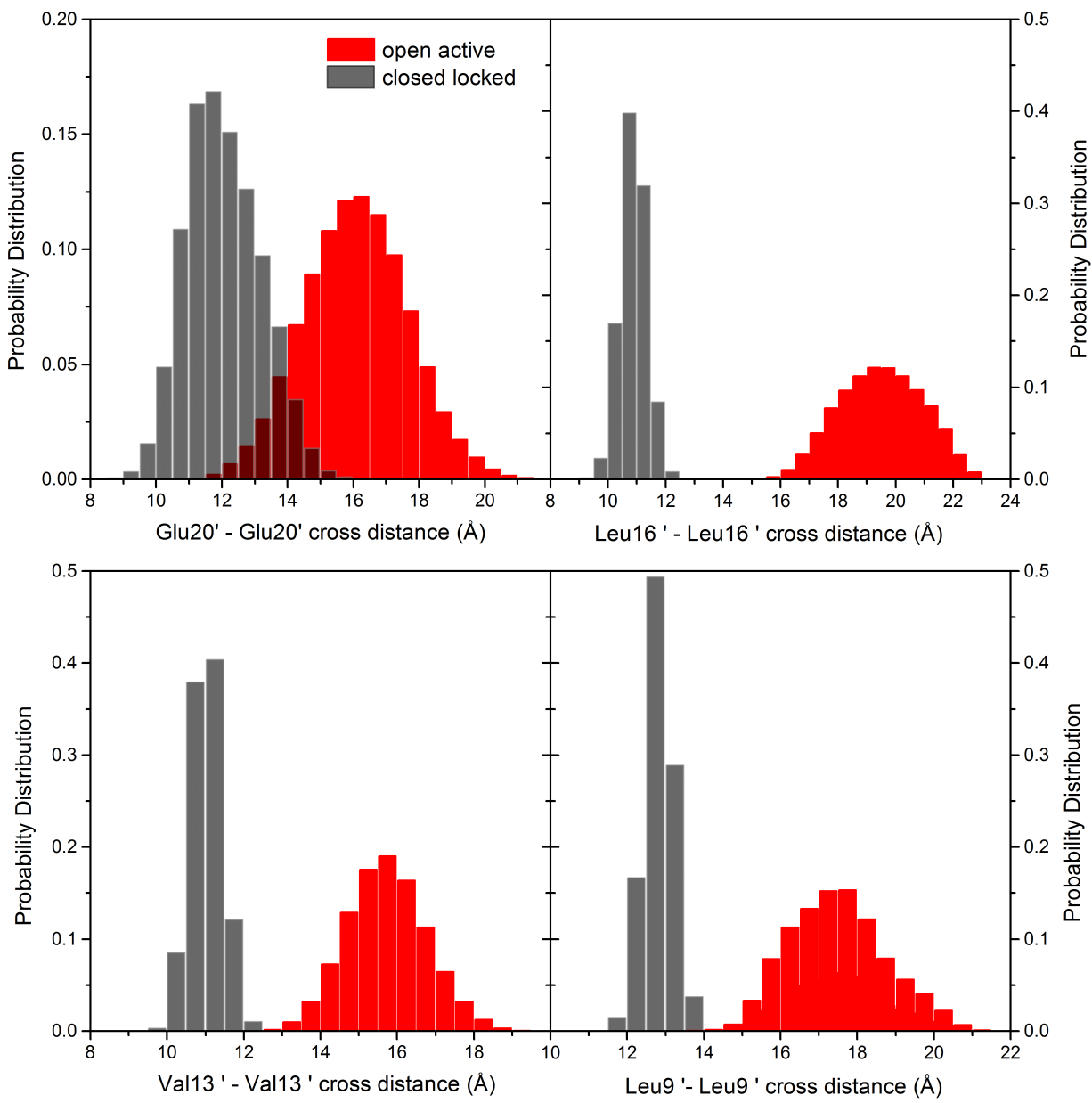

Figure 6: Distributions of crossed distances between pairs of non-adjacent subunits for Leu9', Val13', Leu16' and Glu20'; data collected from the five subunits. Gray bars: closed channel; red bars: open active channel.

in a smaller volume close to the Val13' ring, for all the modeled structures compared in this work.

Focusing only on the comparison between the closed-locked and the open conformations, the number of water molecules in the full channel, $30 \AA$ long, is stationary at $\sim 55$ along the whole simulation of the closed-locked, to be compared with the value of 105 in the open case. In the region Leu9'-Leu16', $10 \AA$ long, the average number of water molecules is about 3 in the closed-locked, and about 30 in the open structure. No re-wetting events are observed, contrary to the putative desensitized structure. $\frac{19}{19}$ 


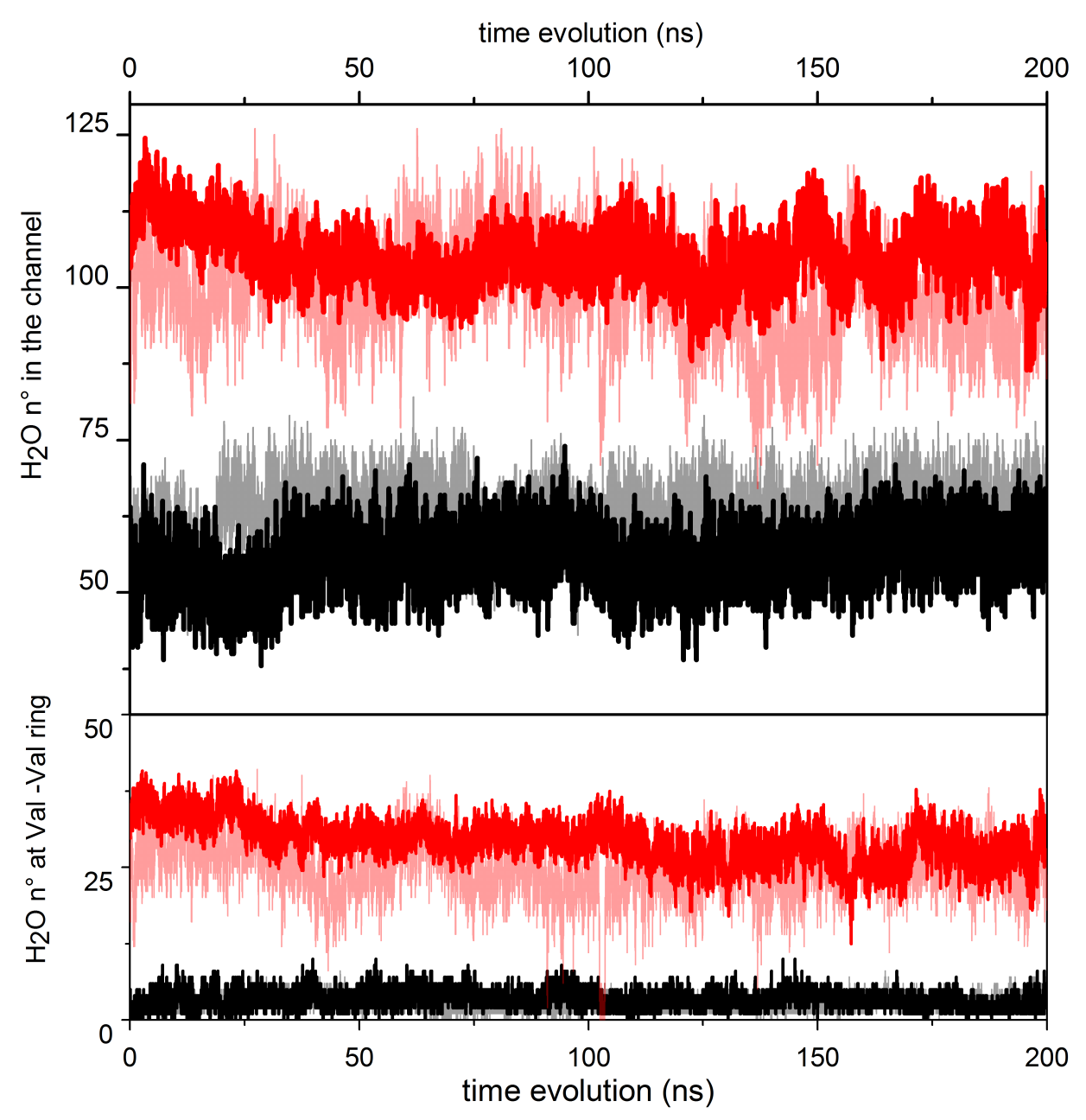

Figure 7: Upper panel: time evolution of water count in the pore lumen lined by the M2 helices. Lower panel: time evolution of water count in a region of $10 \AA$ length, centered at the constriction point 13' (Val246). Black curve: closed-locked; red curve: open active; gray curve: apo-closed; light red curve: apo-open.

\section{Intra protein hydrogen bonding analysis}

Many efforts have been done in the past in finding specific interactions, in particular electrostatic interactions, that could exert a precise control over the gating process. ${ }^{61}$ In the present work, the analysis of the intra protein hydrogen bonding distances (or H-bond distances) points out relevant differences between the closed and the open active structures. In order to mark these differences we start by reporting the bonding pattern characterizing the open structure we proposed in Chiodo et al. ${ }^{18}$ which we re-analyzed here. In such open state, H- 
bond distances are persistently observed in time at the TMD/LBD interface between residues in the principal and complementary subunits (Fig. 8, upper panels). For example, two Hbond distances are present at all times between P4Ser259 in the M2-M3 loop on the principal subunit P4 and P3Asn165 or P3Gly166 in the F loop of the complementary P3(-) subunit; with almost the same persistence in time, we found a H-bond distance between P1Ser259 in the M2-M3 loop in P1 and P5Gly166 in the F loop in P5(-); between P3Ala257 in the M2-M3 loop of P3 and P2Glu167 in the loop F in P2(-); four H-bond distances between P3Asp260 in the M2-M3 loop of P3 and P2Arg199 in the $\beta 1-\beta 2$ in P2(-). A H-bond distance is also found between P3Thr258 and P3Met255 in the M2-M3 loop in P3 and P2Tyr204 in the M1 helix of P2(-); P2Ser259 in the M2-M3 loop of P2 and P1Tyr204 in the M1 helix of P1(-). Seven intra-subunits H-bond distances are also quite often observed in the open state, as in the case of residues at $\beta 1$ vs. residues in the $\mathrm{F}$ loop regions, in almost all subunits.

The LBD/TMD inter-subunit H-bond distances found in the open case are absent in the closed-locked structure. The inter-subunit are replaced by a larger number of intra-subunit $\mathrm{H}$-bond distances, in all subunits. In particular in $\beta 1-\mathrm{F}$ loop pairs, residues in the $\beta 1$ and in the F loop are at a smaller relative distance, due to the outward protrusion of the C-loop capping the conotoxin (Fig. 8, central panels, for the P2 subunit). Visual inspection of the structure suggests that this is also the reason why M2-M3/F loop pairs are too far away to form hydrogen bonds as in the open case.

Moreover, in the open form, H-bond distances are also present in the TMD domain (Fig. 8. lower panels) among different helices of the same or even different subunits. This is the case of P4Ser230 and P3Ser230 or P4Ser235 and P3Glu232 (M2 helices in P4-P3(-)). H-bond distances are observed between: P4Gln267 and P4Ser326 (M3-M4 helices in P4); P2Tyr268 and P2Ser217 (M1-M3 helices in P2), P1Ser 217 and P1Tyr268 (M1-M3 helices in P1). In particular, H-bond distances are persistently observed between the helices M2 and M1 of principal and complementary subunits; between P3Ala252 in P3 and P2Tyr204 in P2(-); between P2Ala180 in P2 and P1Tyr204 in P1(-). Remarkably, these interactions are absent 

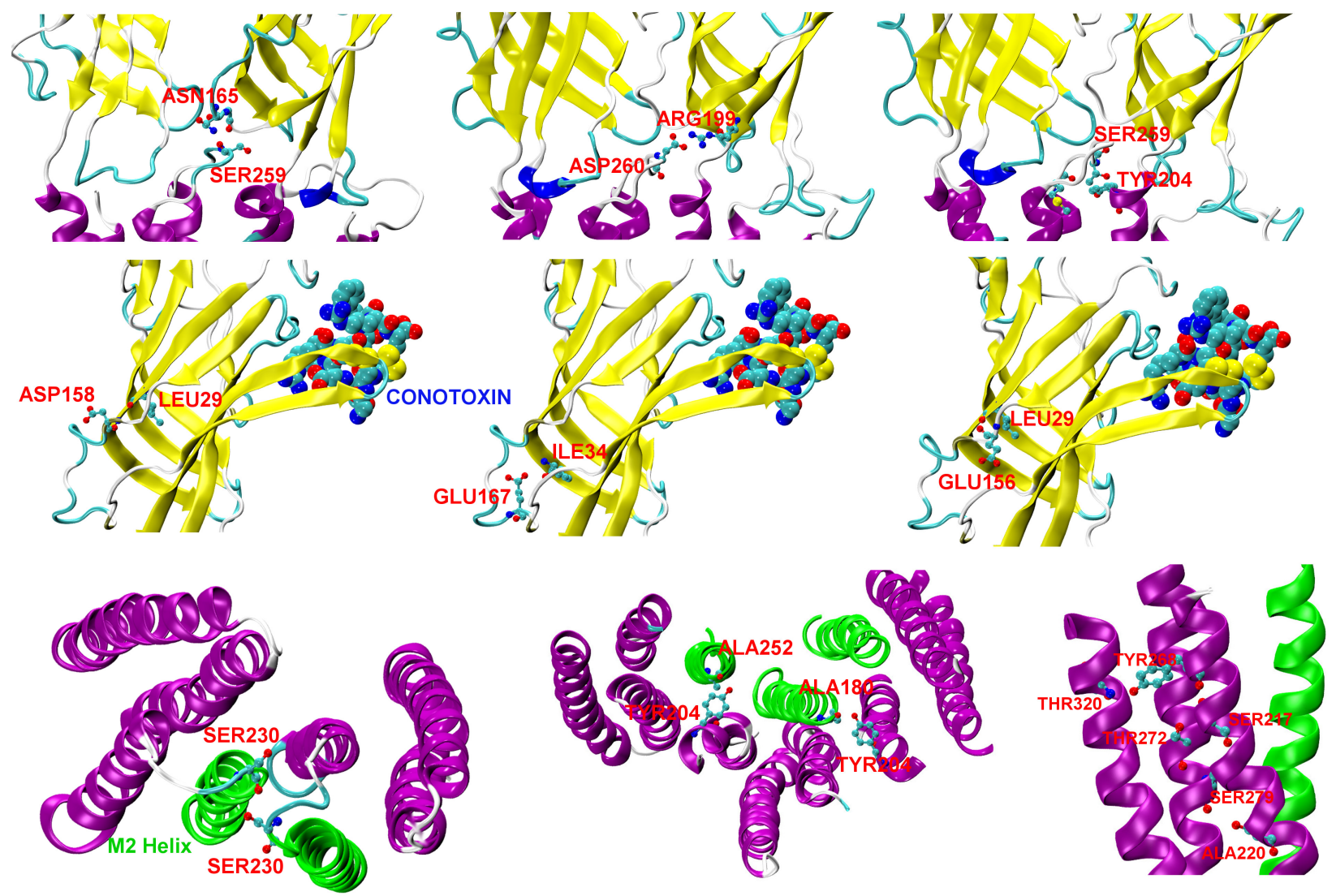

Figure 8: Upper row: selected residues (in ball and stick) in suitable H-bond distances in the open form, at the LBD/TMD interface. Left panel): P4Ser259 (M2-M3)-P3Asn165 (F-loop) in P4-P3(-); central panel) P3Asp260 (M2-M3)-P2Arg199 ( $\beta 10)$ in P3-P2(-); right panel): P2Ser259 (M2-M3)-P1Tyr204 (M1) in P2-P1(-). Central row: selected residues in intrasubunit H-bond distances in the closed form, subunit P2. Left panel): P2Leu29 (F-loop)P2Asp158 ( $\beta 1)$; central panel): P2Glu167 (F-loop)-P2Ile34 ( $\beta 1)$; right panel): P2Glu156 (F-loop)-P2Leu29( $\beta 1)$. Lower row: selected residues in H-bond distances in the TMD M1M4 helices in the open (left and central panels) and in the closed-locked forms (right panel). Green helices: M2; magenta helices: M1, M3, M4. Left panel): P4Ser230-P3Ser230 in the M2 helices P4-P3(-); central panel): P3Ala252-P2Tyr204 in P3-P2(-) and P2Ala180-P1Tyr204 in P2-P1(-); right panel): P1Thr320-P1Tyr268 in the M4 and M3 helices in P1, respectively; P1Ser217-P1Thr272 and P1Ala220-P1Ser279 in the M1 and M3 helices in P1, respectively. The protein is in cartoon and colored according the secondary structure. Conotoxin in the ligand pocket is also shown in vdW representation.

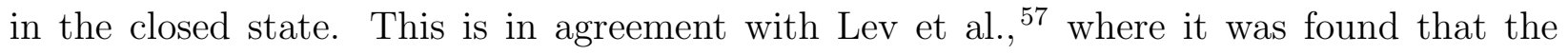
M2-M1(-) bond is a good variable defining pore gating, "undergoing a sizable change that involves loss of helical contacts between subunits" in the open-to-close transition. 
In place of inter-subunit pairs, in the closed state we observe the presence of more intrasubunit H-bond distances between M1-M4 TMD helices with respect the open form (Fig. 8. lower right panel). H-bond distances are found: between P1Ala220 and P1Ser279, P1Ser217 and P1Thr272 in the M1-M3 helices in P1; between P1Thr320 and P1Tyr268 in the M4M3 helices of subunit P1; P3Thr320 and P3Tyr268 in the M4-M3 helices in P3; between P4Thr320 and P4Tyr268 in the M4-M3 of subunit P4; P5Thr320 and P5Tyr268 in the M4-M3 helices in P5.

These results suggest that the open-to-closed transition could be described in terms of the evolution of a network of key hydrogen bonding interactions. In particular, the comparison of the bond pattern between the closed-locked and the open structure points out that subunits communication, both at the interface and in the TMD region, is required for function (binding-to-gating and ion translocation), while it is completely lost in the inactive state.

\section{The apo conformations}

We now report results for the two apo conformations we simulated in this work starting from the closed-locked structure here generated and from the open conformation in $\frac{18}{}$. The differences between these two structures are already evident from the time evolution of the RMSDs with respect to their starting structures (see Figure S2 in Supplementary Material, central and lower panels). In the apo-closed form, the TMD RMSD averaged over the five subunits reaches a stationary value around $1.5 \AA$ after the first $100 \mathrm{~ns}$ of the simulation. The RMSD of the LBD in subunits P4 (blue) and P5 (yellow) appear still increasing on the time scale simulated. We believe that this rise in conformational freedom comes from loops of the binding site that are not bound to the conotoxin molecule anymore, and are thus free to explore more conformations. Indeed, while in subunit P4, the increase is mostly brought about by motions of the $\alpha 1 \beta 1$ and F loops, in subunit P5 is mostly contributed by motions of the C loop, as also testified by the corresponding RMSFs profiles in Figure S3 
(dark yellow curve). These loops are tightly bound to conotoxin when this is present, while there are free to fluctuate in the apo system. This observation is in agreement with results from another MD simulation of a model of the $\alpha 7$ LBD bound to $\alpha$-conotoxin ImI $\cdot \underline{68} \mathrm{~A}$ tight correspondence between the RMDS increasing of one subunit and the higher peak of the F loop in the corresponding RMSF profile is observed in the apo form (see cyan curve in Figure $3 \mathrm{~A}$ and $3 \mathrm{C}$ in $\frac{68}{6}$ ). Something similar is also observed in ${ }^{76}$ where a model of the LBD of the chicken $\alpha 7$ is simulated both bound to cobratoxin and in the apo form on the $60 \mathrm{~ns}$ time scale (see orange curve in Figure $3 \mathrm{~A}$ and $3 \mathrm{C}$ in ${ }^{76}$ for the apo conformation).

As for the apo-open form, the TMD RMSDs appear stationary (below $2 \AA$ ) over the last 200 ns time interval, with the exception of the P2 subunit (red) where the local M3-M4 motion mostly contribute to the RMSD behavior. In the LBD, the non stationary behavior of the P4 subunit (blue) is contributed by local motions of the $\beta 2 \beta 3$ and F loops, as shown in the RMSFs profiles in Figure S3. The peak amplitude of the F-loop is higher than in the case of proteins bound to ligands and also with respect to the apo-closed case.

The ligand pocket conformation in the apo structures was investigated by describing the behavior of the C-loop at the orthosteric site. Distributions of the quantity $d_{C}^{\text {intra }}$ are shown in Figure S4 in Supplementary Material. In the apo systems, the distributions cover values assumed by the corresponding ligand-bound proteins. Moreover, while in the apoopen protein the distribution is shifted towards values proper of the closed-locked form (i.e. the $\mathrm{C}$ loop can be at larger distance from the protein axis), in the apo-closed case it shifts to values proper of the open conformation (i.e. the $\mathrm{C}$ loop can be at shorter distance from the protein axis). The variability of conformations assumed by the $\mathrm{C}$ loop is reflected by the high RMSFs peaks shown in Figure S3 and contributes to the RMSD drifting shown in Figure S2.

As for the water occupancy of the pore, results in Fig. 7 reveal that in the apo-open structure there are larger fluctuations with respect to the epibatidine-bound one, while the apo-closed structure is mostly similar to the conotoxin-bound one. Indeed, along the apo- 

example in the time-intervals $80-100$ ns and $125-150$ ns. Remarkably, in the 102-104 ns interval the water content drops to zero (see Fig. 7, lower panel). The pore profiles (Fig. 9) are superimposed in the closed-locked (black curve) and its apo (grey curve) form, while the apo-open (light red curve) is clearly moving apart the starting holo protein (red curve), in the hundred of nanoseconds time scale. With respect to the open active structure, the pore widens at the intracellular side, in the range between Gly-2' and Thr6', while shrinks in the portion between Thr6 ${ }^{\prime}$ and Glu20'.

The pore profile calculated in a representative snapshot, in the time interval at which water drops to zero (at $102 \mathrm{~ns}$ ), is also shown (Fig. 9, magenta curve). This profile is narrower that the average (light red curve) over the full trajectory, in particular in the region around $\mathrm{Thr} 6^{\prime}$, and in the region $20^{\prime}-13^{\prime}$. At the two minima, the pore radius in this particular apo-open conformation is about $3 \AA$. We do not observe a constriction at Leu9' as in closed structures and apo-GluCl. However, at the intracellular side, the profile is wider than the average, approaching values of the closed states. Overall, the profile resembles the one of Torpedo, also shown in Fig. 9 .

In Fig. 10 a representation of the pore through the molecular structure is shown as a dot surface, along with the pore residues at the observed constriction regions. With respect to the open active state, the reduction of the pore radius at Thr6 is evident.

This peculiar feature of the apo-open structure is confirmed by the distributions of crossed distances in the hydrophobic rings (see Figure S6 in Supplementary Material, for Thr6', Leu9', Val13', Leu16'). The distributions in the apo case become bimodal. This structural variability is expected in an apo conformation,, 4159 and indicates the coexistence of many different conformations. In the case of the Val13' rings, the distances values approach the ones in the closed-locked case. Of particular interest is the distribution of the distances between non-adjacent Thr6' residues. The analysis over the short segment of the trajectory in which de-wetting is observed (102-104 ns) confirms that the Thr6 $6^{\prime}$ crossed distances explore typical 


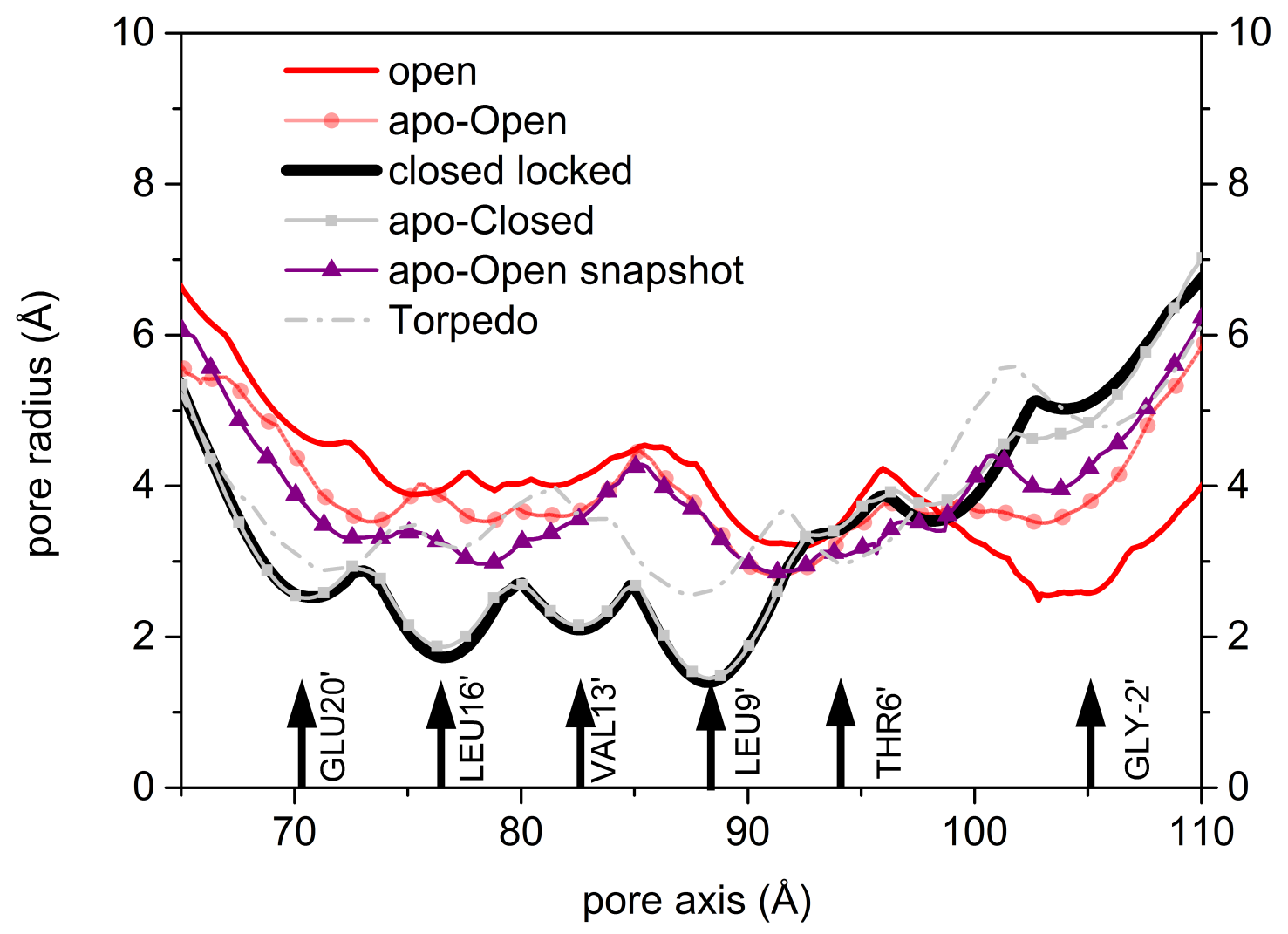

Figure 9: Pore radius profiles along the channel axis in the TMD region, in the apo-closed (gray curve) and apo-open (light red curve) conformations compared with the ones in the open/closed-locked state (red and black curves). Pore profiles are calculated, with HOLE, $\underline{67}$ on structures averaged over the last $200 \mathrm{~ns}$ of the trajectories. The curve in magenta represents the profile in the apo-open in a frame selected in the time interval in which the water content drops to zero (102 ns, see Figure 7, main text). The black arrows, from left to right, indicate the positions of relevant pore-lining residues in the $\alpha 7$.

values found in the closed-locked conformation, even more that the $13^{\prime}$ ring.

As already suggested in procaryotic proteins, ${ }^{77}$ dehydration cannot be considered the only driving force behind pore closure. We performed an analysis of the global quaternary and ternary structural descriptors along the apo-open simulation. Results suggest that removing the ligand induces protein relaxation towards a closed-pore conformation in the time-window observed, although the transition is not complete. Indeed, in the apo-open conformation the global twist, shown in Figure S7 in Supplementary Material, upper left panel, is clearly 
Figure 10: Dot surface of the open (left panel) and apo-open (right panel) channel pores, the latter in a representative snapshot in the time interval in which the pore water content drops to zero (see Figure 7, main text, in the 100-104 ns range). Residues at constriction points are highlighted and colored in orange (from bottom to top, Gly-2', Thr6', Val13', Glu20'). Protein, residues and surface representation as in Figure 5, main text. Constriction points are present in the LBD due to loop conformations from the other subunits (not represented here) extending toward the center of the channel, thus shrinking the pore size.

peaked at intermediate values with respect to the open and closed-locked structures. The blooming distances distribution is shown in Figure S7, upper right panel. Values for both the open and closed-locked conformations were sampled in time. It should be mentioned, however, that these values were found quite similar in the open and closed-locked structures, in a broad range.

Yet the change in the twist angle is not enough to trigger a tangential motion of the M2 helices (see in Figure S7, lower right panel, in Supplementary Material). This is in agreement 
with what reported in $\frac{58}{58}$ on the comparison of $\mathrm{GluCl}$ with and without ivermectin. However, the twist induces the M2 helices polar tilting in the proper direction, as shown in Figure S7, lower left panel, in Supplementary Material.

A change occurs in the direction of closure also at the LBD/TMD interface. Results are shown in Figure S8 in Supplementary Material. We observe that: i) for the Cys-loop/M2M3 distance the distribution is bimodal in the apo-open case, with two peaks in the range of values for open and closed structures; ii) the M2-M3 loop/ $\beta 1$ - $\beta 2$ distance clearly shifts towards the value in the closed state or even beyond.

At variance, in the apo-closed no remarkable change is observed with respect the conotoxin bound structure, besides the higher peak amplitude of the C-loop RMSFs in the absence

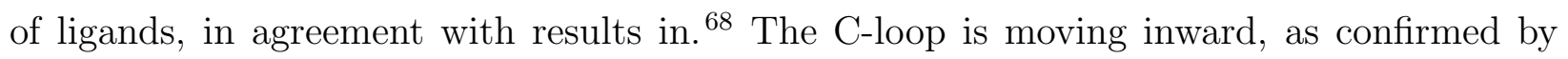
the distribution of the distance of the C-loop tip from the backwall in the LBD (see Figure S4 in Supplementary Material, lower panel, light gray curve). On the simulated time scale, pore structure and water behavior of apo-closed structure are most similar to those of the closed-locked one, bound to toxins; moreover, no re-wetting events are observed, as in a putative desensitized structure. $\frac{19}{}$

\section{Discussion}

The different states along the activation cycle of nicotinic receptors are generally described based on results of electrophysiology experiments: open (conductive, active with bound agonists), closed-locked (in presence of antagonists), resting (or apo, non conductive, without ligands), and desensitized (inactive, even in presence of bound agonists). The determination of structural and dynamical descriptors to unambiguously distinguish these states is however still a largely debated and unsolved issue, in particular for the inactive states. Indeed, no clear correspondence between the physiological observations and simple structural criteria is apparent, with possibly the single exception of the C loop opening in the LBD. $\stackrel{37}{\text { However, }}$ 

beta-erythroidine (DHE), ${ }^{78}$ which is an antagonist, but so small that the C loop does not open more than in presence of an agonist.

The presence of a hydrated, ion-conductive TMD pore is generally considered a marker for the active state. The situation for non-conductive states (closed-locked, resting and desensitized) is quite more complex, since they all show a fully or partially de-hydrated pore. Structural markers might only be identified in fine details of the TMD (relative orientation of the helices and inter-helices loops flexibility), and possibly at the TMD-LBD interface, to reconcile a closed channel structure with different conformations of the LBD. Most difficulties in defining the structural determinants of non-conductive states stem from the paucity of closed-locked, antagonist-bound experimental structures. Only recently a member of the pLGICs family (the anion selective glycine binding channel) has been crystallized in a closedlocked structure, complexed with strychnine. 20

In the present work, we investigated different structural models of non-conductive states for the human $\alpha 7$ channel. We first built a homology model of a closed-locked conformation in complex with conotoxin. Then, using extensive MD simulations, we relaxed the structure and assessed its stability. From the analysis of the simulated trajectory, we identified the structural properties that characterize this conformation as closed, using as reference available experimental data for pLGICs in closed-locked, $\stackrel{20}{a}$ apo resting $2[26$ and desensitized states,,$\sqrt{2325}$ as well as our previously published open-state ${ }^{18}$ and desensitized $\alpha 7$ structures. 19

The model structure is stable on the hundreds of nanosecond time scale, as revealed by the time behavior of the $\mathrm{C} \alpha \mathrm{RMSD}$ with respect to the starting point of the simulation. Features of the $\mathrm{C} \alpha$ RMSFs profile along the protein chain are in good agreement with those reported in literature for a $\alpha 7$ structure modeled on the Torpedo in the closed/desensitized state. 11

We analyzed quaternary and tertiary structural descriptors, invoked to describe functional changes in nAChRs in response to agonist/antagonist binding. Specifically, we moni- 
tored the global twist angle and the blooming of the LBD. The twist angle value is expected to increase during the open-to-close transition, as it describes a rotation of the LBD and TMD in opposite direction around the pore axis, which closes the channel. 52.55 As for the blooming, it has been reported that, with respect to open conformation, a closed channel should expand the LBD radially with respect the channel axis. $.2130 \mid 41 / 57$ [59 A comparison of the values of these two descriptors in our model structure and in our open active model $l^{18}$ points out that the former is a closed conformation. This conclusion is also reinforced by the observation of consistent conformational changes at the LBD/TMD interface and M2 helices tilting.

We also monitored the pore geometry, the time-dependence of its hydration, and the local details of the hydrophobic rings. The pore profile is very similar to the one observed in the strychnine-bound, closed structure of the Gly receptor. ${ }^{20}$ No pore re-wetting is occurring at any time, contrary to what observed, for example, in our simulation of a putative desensitized state on the same time scale. $\frac{19}{19}$ Moreover, analysis of intra-protein hydrogen bonding distances revealed the absence of inter-subunit interactions at the LBD-TMD interface and within the TMD, which are otherwise necessary to establish the communication among subunits underlying ligand-triggered channel gating. ${ }^{[79}$ Based on all these observations, we propose this structure as representative of the physiologically inactive state of the human $\alpha 7$ nicotinic receptor.

We then simulated two more structures, with the purpose of observing spontaneous relaxation of the channel from ligand-complexed states, after removing the drug. The first structure is obtained from the conotoxin-bound, closed conformation presented in this work, while the second from the epibatidine-bound, active conformation we proposed earlier. $\frac{18}{18}$

Our results showed that in the apo-closed structure, during the whole simulated trajectory, the pore remains similar to the one of the original structure from which apo-closed is derived, consistent with a high stability of the closed conformation bound to conotoxin. Moreover, this similarity is also testified by the RMSD behavior of the TMD in the apo- 
At variance, the apo structure from the active, epibatidine-bound channel displays more heterogeneous features. During the simulation, it spontaneously evolves towards a nonconductive conformation, as evidenced by the analysis of the pore hydration. Several dewetting events occur along the simulation. High conformational heterogeneity is observed at the channel hydrophobic girdles and Thr6', especially when compared to the closed-locked trajectory, where the reduced flexibility could be associated to a loss of physiological function. According to our results, it seems that Thr6' is also regulating the pore hydration/dehyration mechanism. Visual inspection of the apo-open trajectory reveals that the shrinking of the Thr6 ${ }^{\prime}$ ring causes the de-wetting observed in the water time series shown in Fig. 7. In Fig. 11, two water configurations in the pore are shown: i) a case of zero water content in the region around $13^{\prime}$ (left panel), and ii) at subsequent time, when water fills again the pore from the bottom (right panel). Such water arrangement is reminiscent of what observed in liquid-vapor transition which occurs in hydrophobic nanopores (hydrophobic gating). $\frac{80}{8}$

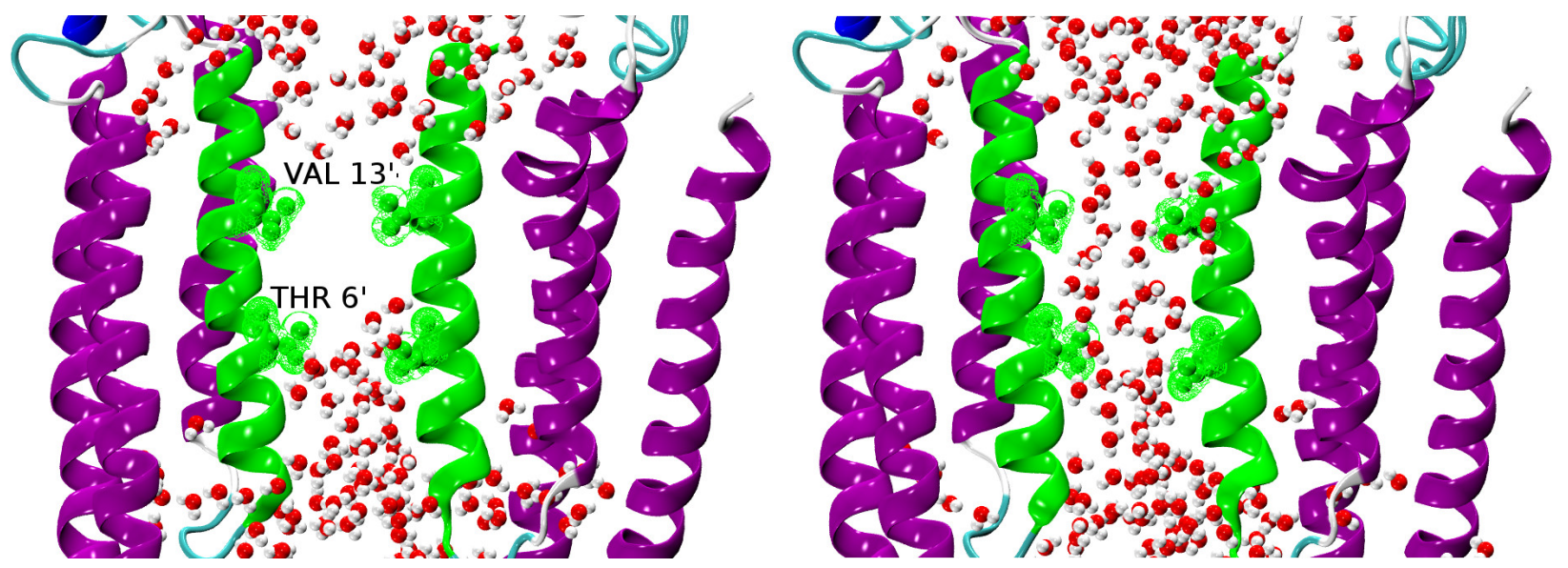

Figure 11: Water configuration in two representative snapshots in the portion 100-104 ns of the apo-open trajectory (see in Figure 7, main text). TMD of the subunits P1, P3 are shown in carton, colored coded according the secondary structure. M2 helices are highlighted in green. Water molecules are in vdW representation. Thr6 ${ }^{\prime}$ and Val13' ${ }^{\prime}$ in subunits P1, P3 are represented in $\mathrm{vdW}$ surrounded by a mesh, in green. Left panel: at $102 \mathrm{~ns}$; right panel: few frames later, after water molecules fill the pore. 
A key role for the Thr6 $6^{\prime}$ ring in maintaining a conductive $\alpha 7$ structure was apparent from our simulations of the active state model of the receptor. $\stackrel{18}{18}$ In that study, we used the open GluCl structure ${ }^{31}$ as template, removing the positive allosteric modulator ivermectin present in the original structure. To avoid the pore collapse after ivermectin deletion, observed

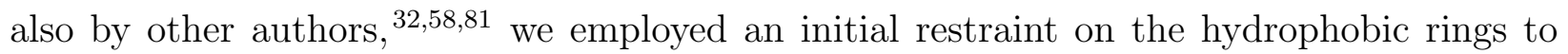
pull apart the hydrophobic sidechains, thus creating a water-accessible volume in the pore. As a consequence, water molecules were able to fill the pore and a hydrated, open pore conformation was stably observed during the subsequent extended simulations we performed shutting off the restraint. Water was found to play an active role in stabilizing the pore from collapse successive to the restraint removal, by strongly coordinating to the Thr $6^{\prime}$ hydrophilic rings and by preventing, in this way, hydrophobic residues to rotate back towards the pore. In support of the importance of polar residues at position $6^{\prime}$ in the ion translocation process, an active role of Ser6 ${ }^{\prime}$ has been inferred from the crystal structure of an open form of GLIC, and confirmed by electrophysiology measurements and MD simulations of native and mutants forms. $\underline{29}$

In summary, according to our analysis, it appears that the apo-open conformation is intermediate between the active and inactive states. This emerges from features at both the LBD and the TMD, as evidenced by the bimodal distributions of key distances at the LBD/TMD interface. For other descriptors, the probability distributions clearly overlap with the closed state. Remarkably, in the portion of the apo-open trajectory when pore closure is observed, all inter-subunit H-bond distances at the TMD/LBD interface, markers of the open active state, are not formed. This conformation is also quite different from the desensitized form we presented recently ${ }^{19}$ for the same channel, thus we suggest it could be associated to the resting apo state of the $\alpha 7$ human receptor. 


\section{Conclusions}

In this work, we provide two new all-atom structural models of the human $\alpha 7$ nicotinic receptor in different states. The first model, which recapitulates the features of a closedlocked state, was generated by homology modeling and relaxed via extensive MD simulations. We used as templates the structure of Aplysia AChBP bound to $\alpha$-conotoxin ImI for the LBD, and the GluCl apo crystal for the TMD. The non-conductive features of the structure were assessed based on the analysis of quaternary and ternary structure descriptors and pore geometry and hydration. This closed-locked conformation and our previous model of the open active conformation bound to the agonist epibatidine provide thermodynamically stable end-points of the gating transition, which, at variance with procaryotic channels, are currently not available for the human channel from high-resolution experimental techniques. Our models could be used to investigate computationally the thermodynamics and kinetics of the conformational cycle using path-sampling algorithms, where the reliability of end-point structures is a fundamental requirement.

In this perspective, in the present work we also destabilized both our ligand-bound structure models and investigated their response on the short time-scale. Indeed, we were interested in capturing any possible early event of the gating transition in the human $\alpha 7$. Remarkably, in the apo structure from the agonist-bound (apo-open), features of a nonconductive conformation such as a reduced pore hydration emerge over few hundreds of nanoseconds. Interestingly, this is consistent with previous claims that the pore hydration is a direct indicator of the channel functional state providing meaningful information already on the sub- microsecond time scale. ${ }^{82}$ We associate the apo-open conformation to the resting-apo form of $\alpha 7$.

In summary, a structural characterization of two non-active states of the human $\alpha 7$ nicotinic receptor is provided, establishing reliable references for the correspondence between structural features and the states of the channel. The availability of structural models for the active and different inactive states of the human nicotinic receptor provides new valuable 
tools to characterize different pLGICs states and to interpret results from experiments or simulations. In particular key interactions at the interface between the extracellular domain and the transmembrane domain are identified, that could be critical to receptor function.

\section{Acknowledgement}

We acknowledge CINECA awards under the ISCRA initiative (grant ID HP10BNVKC9 "IONLGIC"; grant ID HP10C1N72H "CLOSNICO"), the SFI/HEA Irish Center for HighEnd Computing (ICHEC, grant ID ndlif062b "A full atomistic computational study of the active and inactive states of the human 7 nicotinic receptor") and the Poznań Supercomputing and Networking center (PSNC), under the DECI-tier 1 initiative (grant ID 336336 "PRACE-MDNICO"), for the availability of high performance computing resources and support.

\section{Supporting Information Available}

Supporting information are available: Sequence alignment, $\mathrm{C} \alpha$ Root Mean Square Deviation (in $\AA$ ) of individual subunits calculated from the starting conformations (after equilibration); $\mathrm{C} \alpha$ RMSFs (in $\AA$ ) in the individual subunits with respect to the average structure calculated along the final 100 ns of each trajectory; distribution of $d_{C}^{\text {intra }}$ in the closed-locked, open active, apo-open and apo-closed structures; ligand pocket configuration and interaction network; distributions of crossed distances between pairs of non-adjacent subunits; distribution of twist angles, blooming distances, polar and azimutal tilt angles; distributions of the distances between the $\mathrm{C}_{\alpha}$ atom of Pro256 on the M2-M3 loop and the center of mass of the Cys-loop $C_{\alpha}$ atoms.

This material is available free of charge via the Internet at http://pubs .acs.org/. 


\section{Contributions}

TEM, GC, LC, LM conceived and designed the study; LC, GC performed the simulations;

LC, GC, TEM, SG analyzed the data. All authors wrote the paper. 


\section{References}

(1) Gonzalez-Gutierrez, G.; Wanf, Y.; Cymes, G. D.; Tajkhorshid, E.; Grosman, C. Chasing the Open-State Structure of Pentameric Ligand-Gated Ion Channel. J. Gen. Physiol. 2017, 149, 1119-1138.

(2) Wu, Z.; Cheng, H.; Jiang, Y.; Melcher, K.; Xu, H. E. Ion Channels Gated by Acetylcholine and Serotonin: Structures, Biology, and Drug Discovery. Acta Pharmacol. Sin. 2015, 36, 895-907.

(3) daCosta, C. J. B.; Baenziger, J. E. Gating of Pentameric Ligand-Gated Ion Channels: Structural Insights and Ambiguities. Structure 2013, 21, 1271-1283.

(4) Nys, M.; Kesters, D.; Ulens, C. Structural Insights into Cys-Loop Receptor Function and Ligand Recognition. Biochem. Pharm. 2013, 86, 1042-1053.

(5) Bouzat, C. New Insights into the Structural Bases of Activation of Cys-Loop Receptors. J. Physiol. 2012, 106, 23-33.

(6) Corringer, P. J.; Poitevin, F.; Prevost, M. S.; Sauguet, L.; Delarue, M.; Changeux, J. P. Structure and Pharmacology of Pentameric Receptor Channels: from Bacteria to Brain. Structure 2012, 20, 941-56.

(7) Changeux, J. P. The Nicotinic Acetylcholine Receptor: the Founding Father of the Pentameric Ligand-Gated Ion Channel Superfamily. J. Biol. Chem. 2012, 287, 4020740215.

(8) Hilf, R. J.; Dutzler, R. A Prokaryotic Perspective on Pentameric Ligand-Gated Ion Channel Structure. Curr. Op. Struct. Biol. 2009, 19, 418-424.

(9) Corringer, P. J.; Baaden, M.; Bocquet, N.; Delarue, M.; Dufresne, V.; Nury, H.; Prevost, M. S.; Van Renterghem, C. Atomic Structure and Dynamics of Pentameric Ligand- 
Gated Ion Channels: New Insight from Bacterial Homologues. J. Physiol. 2008, 4, $565-572$.

(10) Amiri, S.; Thai, K.; Beckstein, O.; Biggin, P. C.; Sansom, M. S. P. The $\alpha 7$ Nicotinic Acetylcholine Receptor: Molecular Modelling, Electrostatics, and Energetics. Mol. Membr. Biol. 2005, 22, 151-162.

(11) Law, R. J.; Henchman, R. H.; McCammon, J. A. A Gating Mechanism Proposed from a Simulation of a Human $\alpha 7$ Nicotinic Acetylcholine Receptor. Proc. Natl. Acad. Sci. USA 2005, 102, 6813-6818.

(12) Cheng, X.; Ivanov, I.; Wang, H.; Sine, S. M.; McCammon, J. A. Nanosecond-Timescale Conformational Dynamics of the Human $\alpha 7$ Nicotinic Acetylcholine Receptor. Biophys. J. 2007, 93, 2622-2634.

(13) Haddadian, E. J.; Cheng, M. H.; Coalson, R. D.; Xu, Y.; Tang, P. In Silico Models for the Human $\alpha 4 \beta 2$ Nicotinic Acetylcholine Receptor. J. Phys. Chem. B 2008, 112, 13981-13990.

(14) Grazioso, G.; Cavalli, A.; Amici, M. D.; Recanatini, M.; Micheli, C. D. Alpha7 Nicotinic Acetylcholine Receptor Agonists: Prediction of their Binding Affinity through a Molecular Mechanics Poisson Boltzmann Surface Area Approach. J. Comput. Chem. 2008, 29, 2593-2602.

(15) Mowrey, D.; Haddadian, E. J.; Liu, L. T.; Willenbring, D.; Xu, Y.; Tang, P. Unresponsive Correlated Motion in $\alpha 7 \mathrm{nAChR}$ to Halothane Binding Explains Its Functional Insensitivity to Volatile Anesthetics. J. Phys. Chem. B 2010, 114, 7649-7655.

(16) Cheng, M. H.; Coalson, R. D. Energetics and Ion Permeation Characteristics in a Glutamate-Gated Chloride (GluCl) Receptor Channel. J. Phys. Chem. B 2012, 116, $13637-13643$. 
(17) Yu, R.; Hurdiss, E.; Greiner, T.; Lape, R.; Sivilotti, L.; Biggin, P. C. Agonist and Antagonist Binding in Human Glycine Receptors. Biochemistry 2014, 53, 6041-6051.

(18) Chiodo, L.; Malliavin, T. E.; Maragliano, L.; Cottone, G.; Ciccotti, G. A Structural Model of the Human $\alpha 7$ Nicotinic Receptor in an Open Conformation. PloS ONE 2015, 10, e0133011.

(19) Chiodo, L.; Malliavin, T. E.; Maragliano, L.; Cottone, G. A Possible Desensitized State Conformation of the Human $\alpha 7$ Nicotinic Receptor: A Molecular Dynamics Study. Biophys. Chem. 2017, 229, 99-109.

(20) Huang, X.; Chen, H.; Michelsen, K.; Schneider, S.; Shaffer, P. L. Crystal Structure of Human Glycine Receptor- $\alpha 3$ Bound to Antagonist Strychnine. Nature 2015, 526, $277-280$.

(21) Dellisanti, C. D.; Ghosh, B.; Hanson, S. M.; Raspanti, J. M.; Grant, V. A.; Diarra, G. M.; Schuh, A. M.; Satyshur, K.; Klug, C. S.; Czajkowski, C. Site-Directed Spin Labeling Reveals Pentameric Ligand-Gated Ion Channel Gating Motions. PLoS Biol. 2013, 11, e1001714.

(22) Pedersen, S. E. Nicotinic Receptors, The Receptors; Lester RAJ, 2014; Vol. 26.

(23) Morales-Perez, C. L.; Noviello, C. M.; Hibbs, R. E. X-ray structure of the human $\alpha 4 \beta 2$ nicotinic receptor. Nature 2016, 538, 411-415.

(24) Miller, P. S.; Aricescu, A. R. Crystal Structure of a Human GABA(A) Receptor. Nature 2014, 512, 270-275.

(25) Basak, S.; Schmandt, N.; Gicheru, Y.; Chakrapani, S. Crystal Structure and Dynamics of a Lipid-Induced Potential Desensitized-State of a Pentameric Ligand-Gated Channel. Elife 2017, 6, e23886. 
(26) Unwin, N. Refined Structure of the Nicotinic Acetylcholine Receptor at $4 \AA$ Resolution. J. Mol. Biol. 2005, 346, 967-989.

(27) Unwin, N. Nicotinic Acetylcholine Receptor and the Structural Basis of Neuromuscular Transmission: Insights From Torpedo Postsynaptic Membranes. Q. Rev. Biophys. 2013, $46,283-322$.

(28) Hilf, R. J.; Dutzler, R. X-Ray Structure of a Prokaryotic Pentameric Ligand-Gated Ion Channel. Nature 2008, 452, 375-379.

(29) Sauguet, L.; Poitevin, F.; Murail, S.; Van Renterghem, C.; MoragaCid, G.; Malherbe, L.; Thompson, A. W.; Koehl, P.; Corringer, P.; Baaden, M.; Delarue, M. Structural Basis for Ion Permeation Mechanism in Pentameric Ligand-Gated Ion Channels. EMBO J. 2013, 32, 728-741.

(30) Althoff, T.; Hibbs, R. E.; Banerjee, S.; Gouaux, E. X-Ray Structures of GluCl in Apo States Reveal a Gating Mechanism of Cys-Loop Receptors. Nature 2014, 512, 333-337.

(31) Hibbs, R.; Gouaux, E. Principles of Activation and Permeation in an Anion-Selective Cys-Loop Receptor. Nature 2011, 474, 54-60.

(32) Martin, N. E.; Malik, S.; Calimet, N.; Changeux, J. P.; Cecchini, M. Un-Gating and Allosteric Modulation of a Pentameric Ligand-Gated Ion Channel Captured by Molecular Dynamics. PLoS Comput. Biol. 2017, 13, e1005784.

(33) Cheng, Q.; Yakel, J. L. The Effect of $\alpha 7$ Nicotinic Receptor Activation on Glutamatergic Transmission in the Hippocampus. Biochem. Pharmacol. 2015, 97, 439-444.

(34) Bouzat, C.; Lasala, M.; Nielsen, B. E.; Corradi, J.; del Carmen Esandi, M. Molecular Function of $\alpha 7$ Nicotinic Receptors as Drug Targets. J. Physiol. 2018, 596, 1847-1861.

(35) Zhao, Y. The Oncogenic Functions of Nicotinic Acetylcholine Receptors. J. Oncol. 2016, 2016, 9650481. 
(36) Hansen, S. B.; Sulzenbacher, G.; Huxford, T.; Marchot, P.; Taylor, P.; Bourne, Y. Structures of Aplysia AChBP Complexes with Nicotinic Agonists and Antagonists Reveal Distinctive Binding Interfaces and Conformations. EMBO J. 2005, 24, 3635-3646.

(37) Mohammad Hosseini Naveh, Z.; Malliavin, T. E.; Maragliano, L.; Cottone, G.; Ciccotti, G. Conformational Changes in Acetylcholine Binding Protein Investigated by Temperature Accelerated Molecular Dynamics. PLoS ONE 2014, 9, e88555.

(38) McIntosh, J. M.; Yoshikami, D.; Mahe, E.; Nielsen, D. B.; Rivier, J. E.; Gray, W. R.; Olivera, B. M. A Nicotinic Acetylcholine Receptor Ligand of Unique Specificity, AlphaConotoxin ImI. J. Biol. Chem. 1994, 269, 16733-16739.

(39) Taly, A.; Hnin, J.; Changeux, J. P.; Cecchini, M. Allosteric Regulation of Pentameric Ligand-Gated Ion Channels: An Emerging Mechanistic Perspective. Channels 2014, $8,350-360$.

(40) Changeux, J. P. Protein Dynamics and the Allosteric Transitions of Pentameric Receptor Channels. Biophys. Rev. 2014, 6, 311-321.

(41) Cecchini, M.; Changeux, J. P. The Nicotinic Acetylcholine Receptor and Its Prokaryotic Homologues: Structure, Conformational Transitions and Allosteric Modulation. Neuropharmacology 2015, 96, 137-149.

(42) Webb, B.; Sali, A. Protein Structure Modeling with MODELLER. Methods Mol. Biol. $2017,1654,39-54$.

(43) Laskowski, R. A.; Moss, D. S.; Thornton, J. M. Main-Chain Bond Lengths and Bond Angles in Protein Structures. J. Mol. Biol. 1993, 231, 1049-1067.

(44) Chen, V. B.; Arendall, W. B.; Headd, J. J.; Keedy, D. A.; Immormino, R. M.; Kapral, G. J.; Murray, L. W.; Richardson, J. S.; Richardson, D. C. MolProbity: All- 
Atom Structure Validation for Macromolecular Crystallography. Acta Crystallogr. D 2010, $66,12-21$.

(45) Benkert, P.; Tosatto, S. C.; Schomburg, D. QMEAN: A Comprehensive Scoring Function for Model Quality Assessment. Proteins 2008, 71, 261-277.

(46) Bahsford, D.; Karplus, M. pKa of Ionizable Groups in Proteins: Atomic Detail from a Continuum Electrostatic Model. Biochemistry. 1990, 29, 10219-10225.

(47) Anandakrishnan, R.; Aguilar, B.; Onufriev, A. H++ 3.0: Automating pK Prediction and the Preparation of Biomolecular Structures for Atomistic Molecular Modeling and Simulation. Nucleic Acids Res. 2012, 40, 537-541.

(48) Phillips, J. C.; Braun, R.; Wang, W.; Gumbart, J.; Tajkhorshid, E.; Villa, E.; Chipot, C.; Skeel, R. D.; Kalé, L.; Schulten, K. Scalable Molecular Dynamics with NAMD. J. Comput. Chem. 2005, 26, 1781-1802.

(49) Quigley, D.; Probert, M. Langevin Dynamics in Constant Pressure Extended Systems. J. Chem. Phys. 2004, 120, 11432-11441.

(50) Essmann, U.; Perera, L.; Berkowitz, M.; Darden, T.; Lee, H.; Pedersen, L. G. A smooth particle mesh Ewald method. J. Chem. Phys. 1995, 103, 85778593.

(51) Ryckaert, J. P.; Ciccotti, G.; Berendsen, H. J. C. Numerical integration of the cartesian equations of motion of a system with constraints: molecular dynamics of n-alkanes. $J$ Comput Phys 1977, 23, 327341.

(52) Taly, A.; Delarue, M.; Grutter, T.; Nilges, M.; Le Novère, N.; Corringer, P.-J.; Changeux, J. P. Normal Mode Analysis Suggests a Quaternary Twist Model for the Nicotinic Receptor Gating Mechanism. Biophys. J. 2005, 88, 3954-3965. 
(53) Taly, A.; Corringer, P.-J.; Grutter, T.; Prado, L.; Karplus, M.; Changeux, J. P. Implications of the Quaternary Twist Allosteric Model for the Physiology and Pathology of Nicotinic Acetylcholine Receptors. Proc. Natl. Acad. Sci. USA 2006, 103, 16965-16970.

(54) Cheng, X.; Lu, B.; Grant, B.; Law, R. J.; McCammon, J. A. Channel Opening Motion of $\alpha 7$ Nicotinic Acetylcholine Receptor as Suggested by Normal Mode Analysis. J. Mol. Biol. 2006, 355, 310-324.

(55) Samson, A. O.; Levitt, M. Inhibition Mechanism of the Acetylcholine Receptor by Alpha-Neurotoxins as Revealed By Normal-Mode Dynamics. Biochemistry 2008, 47, $4065-4070$.

(56) Liu, X.; Xu, Y.; Li, H.; Wang, X.; Jiang, H.; Barrantes, F. J. Mechanics of Channel Gating of the Nicotinic Acetylcholine Receptor. PLoS Comput. Biol. 2008, 4, e19.

(57) Lev, B.; Murail, S.; Poitevin, F.; Cromer, B. A.; Baaden, M.; Delarue, M.; Allen, T. W. String Method Solution of The Gating Pathways for a Pentameric Ligand Gated Ion Channel. Proc. Natl. Acad. Sci. USA 2017, 114, E4158-E4167.

(58) Calimet, N.; Simoes, M.; Changeux, J. P.; Karplus, M.; Taly, A.; Cecchini, M. A Gating Mechanism of Pentameric Ligand-Gated Ion Channels. Proc. Natl. Acad. Sci. USA 2013, 110, 3987-3996.

(59) Sauguet, L.; Shahsavar, A.; Poitevin, F.; Huon, C.; Menny, A.; kos Nemecz,; Haouz, A.; Changeux, J.-P.; Corringer, P.-J.; Delarue, M. Crystal Structures of a Pentameric Ligand-Gated Ion Channel Provide a Mechanism for Activation. Proc. Natl. Acad. Sci. USA 2014, 111, 966-971.

(60) Cheng, X.; Wang, H.; Grant, B.; Sine, S. M.; McCammon, J. A. Targeted Molecular Dynamics Study of C-Loop Closure and Channel Gating in Nicotinic Receptors. PLoS Comput. Biol. 2006, 2, 1173-1184. 
(61) Xiu, X.; Hanek, A.; Wang, J. A Unified View of the Role of Electrostatic Interactions in Modulating the Gating of Cys Loop Receptors. J. Biol. Chem. 2005, 280, 41655-41666.

(62) Bouzat, C.; Gumilar, F.; Spitzmaul, G.; Wang, H. L.; D.Rayes,; Hansen, S. B.; Taylor, P.; Sine, S. M. Coupling of Agonist Binding to Channel Gating in an ACh-Binding Protein Linked to an Ion Channel. Nature 2004, 430, 896-900.

(63) Bertozzi, C.; Zimmermann, I.; Engeler, S.; Hilf, R. J.; Dutzler, R. Signal Transduction at the Domain Interface of Prokaryotic Pentameric Ligand-Gated Ion Channels. PLoS Biol. 2016, 14, e1002490.

(64) Bocquet, N.; Nury, H.; Baaden, M.; Le Poupon, C.; Changeux, J. P.; Delarue, M.; Corringer, P.-J. X-Ray Structure of a Pentameric Ligand-Gated Ion Channel in an Apparently Open Conformation. Nature 2009, 457, 111-114.

(65) Hilf, R. J.; Dutzler, R. Structure of a Potentially Open State of a Proton-Activated Pentameric Ligand-Gated Ion Channel. Nature 2009, 457, 115-118.

(66) Mowrey, D.; Cheng, M. H.; Liu, L. T.; Willenbring, D.; Lu, X.; Wymore, T.; Xu, Y.; Tang, P. Asymmetric Ligand Binding Facilitates Conformational Transitions in Pentameric Ligand-Gated Ion Channels. J. Am. Chem. Soc. 2013, 135, 2172-2180.

(67) Smart, O. S.; Neduvelil, J. G.; Wang, X.; Wallace, B. A.; Sansom, M. S. P. HOLE: A Program for the Analysis of the Pore Dimensions of Ion Channel Structural Models. J. Mol. Graph. 1996, 14, 354-360.

(68) Yu, R.; Craik, D. J.; Kaas, Q. Blockade of Neuronal $\alpha 7-n A C h R$ by $\alpha$-Conotoxin ImI Explained by Computational Scanning and Energy Calculations. PLoS Comput. Biol. 2011, \%, e1002011.

(69) Lamthanh, H.; Jegou-Matheron, C.; Servent, D.; Mnez, A.; Lancelin, J. M. Minimal conformation of the alpha-conotoxin ImI for the alpha7 neuronal nicotinic acetylcholine 
receptor recognition: correlated CD, NMR and binding studies. FEBS Lett 1999, 454, 293298 .

(70) Quiram, P. A.; Jones, J. J.; Sine, S. M. Pairwise Interactions between Neuronal $\alpha 7$ Acetylcholine Receptors and $\alpha$-Conotoxin ImI. J. Biol. Chem. 1999, 274, 19517-19524.

(71) Tabassum, N.; Yu, R.; Jiang, T. Computational Determination of the Binding Mode of $\alpha$-Conotoxin to Nicotinic Acetylcholine Receptor. J. Ocean Univ. China 2016, 15, $1027-1033$.

(72) Dutertre, S.; Lewis, R. J. Toxin Insights into Nicotinic Acetylcholine Receptor. Biochem. Pharmacol. 2006, 72, 661-670.

(73) Baptista-Hon, D. T.; Deeb, T. Z.; Lambert, J. J.; Peters, J. A.; Hales, T. G. The Minimum M3-M4 Loop Length of Neurotransmitter-Activated Pentameric Receptors Is Critical for the Structural Integrity of Cytoplasmic Portals. J. Biol. Chem. 2013, 288, 21558-21568.

(74) Quiram, P. A.; Sine, S. M. Structural Elements in $\alpha$-Conotoxin ImI Essential for Binding to Neuronal $\alpha 7$ Receptors. J. Biol. Chem. 1998, 273, 11007-11011.

(75) Li, S.-X.; Huang, S.; Bren, N.; Noridomi, K.; Dellisanti, C. D.; Sine, S. M.; Chen, L. Ligand-binding domain of an $\alpha 7$-nicotinic receptor chimera and its complex with agonist. Nat Neurosci. 2011, 14, 1253-1259.

(76) Yi, M.; Jong, H. T.; Zhou, H. X. Spontaneous conformational change and toxin binding in $\alpha 7$ acetylcholine receptor: Insight into channel activation and inhibition. Proc. Natl. Acad. Sci. USA 2008, 105, 82808285.

(77) Zhu, F.; Hummer, G. Pore Opening and Closing of a Pentameric Ligand-Gated Ion Channel. Proc. Natl. Acad. Sci. USA 2010, 107, 19814-19819. 
(78) Shahsavar, A.; Kastrup, J. S.; Nielsen, E.; Kristensen, J. L.; Gajhede, M.; Balle, T. Crystal Structure of Lymnaea Stagnalis AChBP Complexed with the Potent nAChR Antagonist DH $\beta$ E Suggests a Unique Mode of Antagonism. PLoS ONE 2012, 7, e40757.

(79) Changeux, J. P.; Edelstein, S. J. Allosteric Mechanisms of Signal Transduction. Science 2005, 308, 1424-1428.

(80) Aryal, P.; Sansom, M. S. P.; Tucker, S. J. Hydrophobic Gating in Ion Channels. J. Mol. Biol. 2015, 427, 121-130.

(81) Yoluk, O.; Bromstrup, T.; Bertaccini, E. J.; Trudell, J. R.; Lindahl, E. Stabilization of the GluCl Ligand-Gated Ion Channel in the Presence and Absence of Ivermectin. Biophys. J. 2013, 105, 640-647.

(82) Trick, J. L.; Chelvaniththilan, S.; Klesse, G.; Aryal, P.; Wallace, E. J.; Tucker, S. J.; Sansom, M. S. Functional Annotation of Ion Channel Structures by Molecular Simulation. Structure 2016, 24, 2207-2216. 


\section{Graphical TOC Entry}

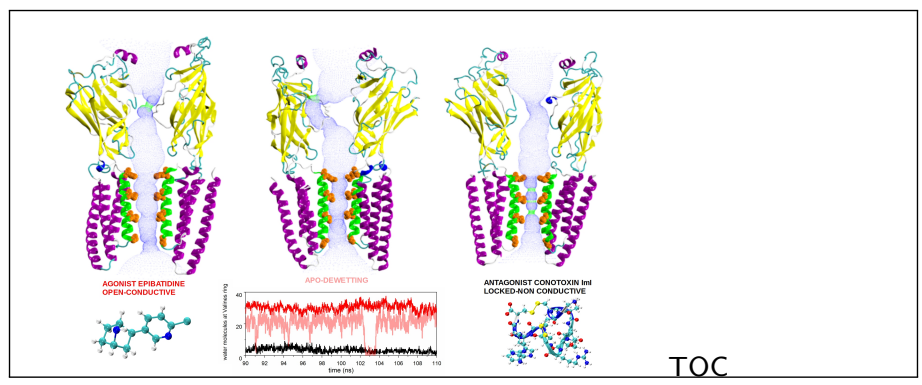

